\title{
Yorkie controls tube length and apical barrier integrity
}

\section{in the developing Drosophila airways}

\author{
Dimitrios K. Papadopoulos ${ }^{1,2}$, Pavel Tomancak ${ }^{1}$, Vasilios Tsarouhas ${ }^{3}$, \\ Christos Samakovlis ${ }^{3,4}$, Elisabeth Knust ${ }^{1, *, *}$ and Kassiani Skouloudaki ${ }^{1, *, \#}$
}

${ }^{1}$ Max-Planck Institute for Molecular Cell Biology and Genetics, 01307 Dresden, Germany

${ }^{2}$ present address: MRC Human Genetics Unit, Institute of Genetics and Molecular Medicine, University of Edinburgh, Edinburgh, UK.

${ }^{3}$ Department of Molecular Biosciences, The Wenner-Gren Institute, Stockholm University, S-10691 Stockholm, Sweden

${ }^{4}$ ECCPS, University of Giessen, Giessen, Germany

*Correspondence: skouloud@mpi-cbg.de and knust@mpi-cbg.de

\#These authors should be considered as joint senior authors.

Running title:

Yorkie controls tracheal expansion and gas filling

Key words:

embryonic trachea, Twinstar/Cofilin, Drosophila, tube expansion, Alas, apical extracellular matrix

Author contributions: DKP, VT and KS, designed and performed the experiments, analyzed the data, prepared figures; PT, CS and EK supervised the project; KS conceived the project and wrote the manuscript with critical input from all authors; all authors critically reviewed the manuscript and approved it for submission. 


\begin{abstract}
Epithelial organ size and shape depend on cell shape changes, cell-matrix communication and apical membrane growth. The Drosophila embryonic tracheal network is an excellent model to study these processes. Here, we show that the transcriptional co-activator of the Hippo pathway, Yorkie (YAP in vertebrates), plays distinct roles in the developing Drosophila airways. Yorkie exerts a cytoplasmic function by binding Drosophila Twinstar, the orthologue of the vertebrate actin-severing protein Cofilin, to regulate F-actin levels and apical cell membrane size, which are required for proper tracheal tube elongation. Second, Yorkie controls water-tightness of tracheal tubes by transcriptional regulation of the enzyme $\delta$-aminolevulinate synthase (Alas). We conclude that Yorkie has a dual role in tracheal development to ensure proper tracheal growth and functionality.
\end{abstract}

\title{
Short Summary
}

This work identified an alternative role of the transcriptional co-activator Yorkie (Yki) in controlling water impermeability and tube size of the developing Drosophila airways. Tracheal impermeability is triggered by Ykimediated transcriptional regulation of $\delta$-aminolevulinate synthase, Alas, whereas tube elongation is controlled by binding of Yki to the actin severing factor Twinstar. 


\section{Introduction}

Regulation of epithelial tube size and integrity depends on various control mechanisms, including cell surface receptors, cytoskeletal and extracellular matrix components, cell polarity and vesicular transport. These distinct cellular mechanisms control the development and maintenance of tube diameter and length, which are key requirements for proper tube function (Beitel and Krasnow, 2000; Iruela-Arispe and Beitel, 2013). Aberrant regulation of any of these components causes human diseases, such as polycystic kidney disease (PKD) (Steinman, 2012), fibrocystic breast disease (Rinaldi et al., 2010), pancreatic cystic neoplasma (Garud and Willingham, 2012) or thyroid nodules (Popoveniuc and Jonklaas, 2012). To understand the biology of epithelial tube formation and functionality, several models have been used, including the well-characterized Drosophila respiratory system, the tracheae. The tracheae form a branched tubular network during the first half of embryogenesis. At mid-embryogenesis, the tubes expand and a transient cable is deposited in the tubular lumen, consisting of a chitinous apical extracellular matrix (aECM), which confers rigidity to the tubes and is responsible for diametric expansion and axial elongation (Forster et al., 2010; Luschnig et al., 2006; Tonning et al., 2005; Wang et al., 2006). Later, endocytosis removes the luminal proteins and the tubes fill with air (Hayashi and Dong, 2017; Tsarouhas et al., 2007). During late embryonic development and after diametrical expansion, tracheal tubes elongate by means of cell shape changes and cell-cell junction rearrangements, but without increasing 
their cell number (Samakovlis et al., 1996). So far, it has been well established that longitudinal growth depends on various cellular components and molecules, including: i) septate junctions (Llimargas et al., 2004; Wang et al., 2006; Wu et al., 2004), ii) the subapical protein Crumbs and the apical cytoskeleton (Dong et al., 2014; Laprise et al., 2006; Laprise et al., 2010; Tonning et al., 2005), and iii) chitin deacetylases and Src kinase levels (Forster and Luschnig, 2012; Luschnig et al., 2006; Nelson et al., 2012; Wang et al., 2006). The transcriptional coactivator Yorkie (Yki) has also been found to be required for proper tracheal tube growth (Robbins et al., 2014), but how a gene, which is mostly implicated in cell proliferation controls growth in a non-proliferating tissue has not been understood.

Yki is a major downstream target of the evolutionarily conserved Hippo signaling pathway, and controls organ size by suppressing proliferation and promoting apoptosis (Halder and Johnson, 2011). Active Hippo signaling phosphorylates Yki, inhibiting its translocation into the nucleus and its transcriptional activity. Upon inactivation of the kinase cascade, nonphosphorylated Yki enters the nucleus and promotes growth by transcriptional activation of genes that inhibit apoptosis, e.g. Death-associated inhibitor of apoptosis 1 (Diap1), or those stimulating proliferation, e.g. Myc.

Since cell proliferation genes are transcriptional targets of $\mathrm{Yki}$ and its conserved vertebrate ortholog YAP (Yes-associated protein), Yki/YAP activity has to be tightly regulated, which is largely mediated by controlling its subcellular localization (nuclear versus cytoplasmic). Several upstream mechanisms are known that control Yki/YAP localization, including its phosphorylation (as part of the evolutionarily conserved Hippo pathway) 
(Fulford et al., 2018), its interaction with tight junction protein complexes that stabilize the cytoplasmic Yki/YAP pool (Skouloudaki and Walz, 2012) and regulate Yki/YAP cytoplasm-to-nucleus translocation in proliferating epithelial tissues (Wang et al., 2011; Zhao et al., 2011), or cellular and extracellular mechanical forces (Totaro et al., 2018).

Here we describe a novel mechanism to control Yorkie cellular localization in a non-proliferating tissue, the tracheal system of Drosophila. We discovered that $\mathrm{Yki}$, which has been shown to control tube length expansion (Robbins et al., 2014) does so by interacting with Twinstar (Tsr), the Drosophila orthologue of vertebrate Cofilin. Tsr keeps Yki apically in tracheal epithelial cells and thereby prevents its nuclear localization. In addition we show that $y k i$ is required for the generation of water impermeable tubes. This function is controlled by the Yki-target gene Alas, which encodes an enzyme that regulates the structure of the apical extracellular matrix (aECM) (Shaik et al., 2012). Thus, our data provide mechanistic evidence how Yki controls tube elongation and impermeability in the tracheae. 


\section{Results}

\section{Yorkie controls tracheal tube length and proper gas-filling}

Drosophila embryos, bearing a deletion of the whole yki locus (Huang et al., 2005) ( $y k i^{B 5}$ loss-of function allele, henceforth referred to as $\left.y k i\right)$, die at late embryonic stages with elongated tracheal tubes (Fig. 1, A and B) (Huang et al., 2005; Robbins et al., 2014). Staining for chitin confirmed that the length of the major tracheal tubes, the dorsal trunks (DTs), of late stage $16 y k i$ mutant embryos is significantly increased compared to wild type (Fig. 1 B). We noticed that beside extended length the DTs fail to clear luminal liquid and do not fill with gas (Fig. 1, C and D). Both phenotypes, i.e. increased tube length and defects in gas filling, were suppressed upon tracheal-specific expression of a cDNA, which encodes a Yki protein C-terminally tagged with V5 (Yki-V5) (Fig. 1, A-D). However, and in contrast to published data (Robbins et al., 2014), we only observed partial rescue of the tube length phenotype of $y k i$ mutants upon tracheal expression of Diap1 [Deathassociated inhibitor of apoptosis 1; also known as thread (thr)], a wellestablished Yki-target gene (Fig. $1 \mathrm{E}$ ). These results indicate that $y k i$ is required to limit tracheal tube length and to promote gas-filling in Drosophila embryos.

\section{Yorkie is required for formation of impermeable tubes}

Liquid impermeability of tracheal tubes has been shown to be controlled by septate junctions (SJ). Loss of SJ components result in leakiness of the tube and impaired gas filling. However, in agreement with previous reports (Robbins et al., 2014), canonical SJ proteins, such as Yurt, 
Coracle, Megatrachea, Varicose, Fasciclin III and Contactin appeared to be localized correctly to SJs in yki mutant tracheae (Fig. 2, A-D and Fig. S1). In agreement with this observation, electron microscopic analysis revealed that the structure of the SJs is not affected in $y k i$ mutant tracheae and showed proper organization of the ladder-like septa similar to wild type embryos (Fig. $2, A, B, D)$. This phenotype was rescued by the tracheal expression of a $y k i$ cDNA (Fig. 2, C, D). Yet, in contrast to recently published data (Robbins et al., 2014), we observed that in approximately $80 \%$ of $y k i$ mutant embryos injected with $10 \mathrm{kDa}$ Dextran to test for the barrier function of SJs, the dye diffused into the lumen of the DTs, which never occurs in wild-type embryos (Fig. 3, A, B and D). Stage 17 embryos expressing a yki transgene fail to fill the tracheal lumen with Dextran (Fig. 3, C, D). This indicates that the tracheal epithelium in yki mutants is not tightly sealed and that this mechanism is yki-dependent. The dye diffused gradually into the tracheal lumen of $y k i$ mutants, with higher amounts observed 240 min after injection (Fig. 3, E-G" and H). This defect is much milder than that observed in embryos mutant for melanotransferrin ( $m t f)$ (Fig. 3, G-G"), a gene encoding a bona-fide SJ component, in which the dye filled the tracheal lumen already after 20min (compare Fig. 3, F-F").

The water-tightness of tracheal tubes is not only ensured by SJs, but also depends on the proper deposition of a chitinous apical extracellular matrix (aECM). Since SJs did not appear impaired in yki mutants, we asked whether loss of $y k i$ affects the secretion of the chitin deacetylases, Serpentine (Serp) and Vermiform (Verm). However, the luminal deposition of Serp and Verm is not perturbed in yki mutants (Fig. 2, A-D and Fig. S2, A-B"), pointing to a 
novel, SJ and luminal matrix deposition-independent function of Yki in the control of tracheal tube impermeability.

\section{Yorkie is required for proper cross-linking of tracheal apical ECM proteins}

Beside proper SJs and secretion of the chitin-modifying enzymes Serp and Verm, formation of an intact apical chitin lattice is necessary to ensure impermeability of tracheal tubes (Shaik et al., 2012). Therefore, we examined the aECM in yki mutant tracheae in more detail. The insect cuticle is an apical extracellular waterproof barrier, deposited by epithelial organs such as the epidermis, the tracheae, the fore- and hindgut, which protects against dehydration and infection and plays a crucial role in organ morphogenesis (Moussian, 2010; Ozturk-Colak et al., 2016). The cuticle is composed of three layers, the outermost envelope, followed by the epicuticle and the procuticle. In the tracheae, the epi- and procuticle form the so-called taenidia, chitinous ridges lining the lumen. These layers are formed by proteins, chitin and lipids. Some of the proteins are cross-linked in order to form an impermeable layer. Three lines of evidence suggested that an impaired function of the $\delta$ aminolevulinate synthase gene Alas (Shaik et al., 2012) is responsible for fluid leakage and gas filling defects in yki mutants. First, ChiP-seq data from Nagaraj et al. showed that Yki binds the regulatory region of the Alas gene in Drosophila imaginal discs (Nagaraj et al., 2012). Second, similarly to yki, Alas is required for air-filling of the tracheal tubes and for the maintenance of tracheal impermeability at later stages of embryogenesis (Shaik et al., 2012). Third, barrier defects observed in Alas mutants occur despite structurally and 
functionally normal SJs (Shaik et al., 2012), similar to $y k i$ mutants. This prompted us to investigate a possible functional relationship between $y k i$ and Alas. Alas mutant tubes display reduced amount of cuticular di-tyrosine bonds, resulting in loss of cuticular impermeability and defects in tracheal airfilling (Fig. 4, A, A', E and G) (Shaik et al., 2012). These phenotypes were strongly suppressed upon tracheal-specific expression of an Alas cDNA (Fig. 4, B, B', E, F and G). Di-tyrosine bonds were also reduced in amount in $y k i$ mutants (Fig. 4, C, C', E and G), suggesting that Alas may act downstream of yki. In fact, gas-filling and trans-epithelial barrier defects were rescued by expression of Alas in tracheal cells of yki mutants (Fig. 4, D, D', E, F and G), while normal tube length was not restored (data not shown). Furthermore, Alas mRNA is almost 2-fold decreased in yki mutants (Fig. $4 \mathrm{H}$ ), corroborating the notion that $\mathrm{Yki}$ is a transcriptional activator of Alas. Interestingly, duox mRNA, which encodes an enzyme that catalyzes the oxidation of two tyrosines to di-tyrosine (Edens et al., 2001) and which is also expressed in developing tracheae (Yao et al., 2017) did not show any significant difference in yki mutants (Fig. $4 \mathrm{H}$ ). This led us to suggest that Yki regulates the dityrosine network through Alas. To test whether Yki is a regulator of di-tyrosine bridges between extracellular proteins in other cuticular organs, we used ptcGal4 to express Yki-V5 (Fig. S3, A -A") or knock it down by yki RNAi (Fig. S3, B-B") along the anterior-posterior (AP) boundary of wing imaginal discs. In Yki-expressing discs, the level of di-tyrosine was considerably increased (Fig. S3 A' arrows, D), whereas di-tyrosine was reduced upon yki knock-down (Fig. S3 B' arrows, D), as compared to the control (Fig. S3, C-C"). 
To summarize, we provide evidence that $\mathrm{Yki}$ acts through transcriptional activation of Alas to regulate extracellular di-tyrosinedependent barrier formation in the tracheae, required for forming waterproof tracheal tubes.

\section{Yorkie controls tracheal tube length by regulating the actin- depolymerizing factor Twinstar/Cofilin}

In contrast to yki mutants, Alas mutants do not exhibit over-elongated DTs (Fig. 4, A, C and Fig. S4, A-C), suggesting that Yki-mediated regulation of tube expansion is independent of Alas. Additionally, the only partial rescue of the tube length in $y k i$ mutants upon Diap1 expression points to an additional, Diap1-independent function of Yki in determining tube size. Several genes are known to restrict tracheal tube length (Hayashi and Kondo, 2018) including those encoding SJ proteins (Zuo et al., 2013), chitin-modifying enzymes (Luschnig et al., 2006; Wang et al., 2006), polarity proteins regulating apical membrane growth (Dong et al., 2014; Laprise et al., 2006) and cytoskeleton proteins and their regulators [recently reviewed in (Hayashi and Kondo, 2018; Ochoa-Espinosa et al., 2012)]. As shown above, yki mutant tracheae bear normal SJs and secrete Verm and Serp normally into the lumen. Localization of the apical determinant Crb and aPKC were apparently not affected in yki mutants (Fig. S5, A-B and A'-B'). Finally, genetic interaction experiments between $y k i$ and Src42a, which encodes a tyrosine kinase, revealed that the two genes act in parallel pathways to regulate tracheal tube length (Robbins et al., 2014). Taken together, our analysis identified a 
function of Yki in the control of tracheal tube length, independent of SJs, luminal matrix deposition and apical polarity.

To unveil the molecular mechanism by which Yki regulates tube length expansion, we immunoprecipitated Yki-V5, expressed in tracheae, and performed mass spectrometry analysis. One of the proteins found to associate with Yki was Twinstar (Tsr), the Drosophila ortholog of vertebrate Cofilin (Table S6 A). Recent studies have implicated Tsr in cell survival, tissue growth and tissue integrity of Drosophila wing imaginal discs by regulating Yki and JNK signaling (Ko et al., 2016). To further confirm that Yki and Tsr interact physically, we examined their interaction using several assays in embryonic tracheae and wing imaginal discs. Overexpressed Yki-V5 coprecipitated Tsr from protein lysates of embryonic tracheae (Fig. 5 A). Further, in-situ proximity ligation assay (PLA) (Soderberg et al., 2006) (Fig. 5 B-C”) and Bimolecular fluorescence complementation (BiFC) (Hu et al., 2002) (Fig. 5 D) both confirmed that the two proteins, when expressed in wing imaginal discs, were found in close proximity. From these results we concluded that Yki and Tsr physically interact to form a protein complex.

To find out whether Tsr and Yki function together to regulate tube size, we analyzed the function of Tsr in the tracheae, using two different alleles, $t s r^{k 05633}$ and $t s r^{N 96 A}(\mathrm{Ng}$ and Luo, 2004; Wahlstrom et al., 2001). Strikingly, tsr mutant embryos exhibited over-elongated tracheal tubes, similar to $y k i$ mutants (Fig. 6 A-C, E and Fig. S7 A-C). This phenotype is due to a specific function of Tsr in the trachea, since tracheal-specific expression of a tsr cDNA by bt/-GAL4 rescued the over-elongated tubes of tsr mutants (Fig. $6 \mathrm{~F}-\mathrm{H}$ ). In contrast to $y k i$, however, tsr mutants showed proper tracheal gas-filling (Fig. 
S7 $\mathrm{D}$ and E) and displayed normal paracellular barrier of the tracheal tubes

(Fig. S7 F and G). These results indicate that tsr functions in tube size control, but not in gas filling.

The similar tracheal phenotypes of tsr and $y k i$ mutants raised the possibility that Yki and Tsr act in the same pathway to control DT length. Strikingly, $y k i$, tsr double mutants had even longer tubes, as compared to those of $y k i$ and tsr single mutants, suggesting that $y k i$ and tsr act in parallel pathways to regulate tube size (Fig. $6 \mathrm{D}$ and E). To determine whether there is any connection between the two pathways to regulate tracheal tube length, we expressed a yki cDNA in the tracheae of tsr mutant embryos. In these embryos tracheal tube length was restored and comparable to that of control embryos (Fig. 6 I). Likewise, expression of a tsr cDNA reversed the yki tube elongation phenotype (Fig. $6 \mathrm{~J}-\mathrm{M}$ ). These data support that tsr and yki act in parallel, yet interconnected pathways.

\section{Tsr regulates Yki nuclear activity}

To determine the functional relationship between $\mathrm{Tsr}$ and $\mathrm{Yki}$ in the developing Drosophila airways, we first asked how they cooperate to control tube length. Interestingly, Yki binding sites were also identified in the regulatory sequences of $t s r$ (Nagaraj et al., 2012), suggesting a transcriptional regulation of tsr by Yki in imaginal discs. However, we did not find any significant alteration in tsr mRNA levels between control and stage $17 \mathrm{yki}$ mutants by quantitative real-time PCR (Fig. $6 \mathrm{~N}$ ), while yki mRNA was undetectable in $y k i$ mutants at this stage (Fig. 6 O). Therefore, we asked whether our inability to detect changes in tsr mRNA levels are owed to 
maternal Yki protein, which can be detected in unfertilized eggs (Fig. S8 A). We undertook a biochemical approach and observed a Yki life time of 11-16 hours in $\mathrm{S} 2$ cells (half-life of $\mathrm{t}_{1 / 2} \approx 3 \mathrm{~h}$ ). Assuming the same life time in embryos suggests the absence of Yki protein (maternal and zygotic) in stage 17 yki mutants, at which tsr transcripts were analyzed (Fig. S8, B-C and Fig. 6, P-R). From this we conclude that $t s r$ is not a transcriptional target of $y k i$ in the embryo (or in the tracheae).

How is then Tsr regulated? Interestingly, in contrast to tsr transcripts, the protein levels were reduced in stage 17 yki mutant embryos. At the same time, we observed a mutual regulation between Yki and Tsr, in that Yki protein is also reduced in tsr mutant embryos (Fig. 6 P-R). To determine whether the Tsr-dependent changes in Yki levels go along with changes in Yki transcriptional activity, we analyzed in vivo the transcription of one if its target genes, Diap1. Diap1 mRNA levels were significantly reduced in $y k i$ mutant embryos but increased about two-fold in $t^{k 05633}$ mutants (Fig. $6 \mathrm{~S}$ ). To determine whether this increase in diap1 transcription is also observed in the tracheae, we used the $y k i$ transcriptional reporter, Diap1-lacZ-NLS (Ryoo et al., 2002). In fact, the reporter showed stronger expression in tracheal cells of tsr mutant embryos (Fig. $7 \mathrm{~A}$ and $\mathrm{B}$ ).

Yki activity is mostly controlled by phosphorylation of S168 (in Yki) and S127/S381 (in Yki/YAP). Phosphorylated Yki/YAP is retained in the cytoplasm, while non-phosphorylated Yki/YAP can enter the nucleus and activate transcription of its target genes (Oh and Irvine, 2011). Using the phosphate-binding tag method (Kinoshita et al., 2006) to capture the phosphorylated form of Yki in embryonic protein lysates, we could not draw 
any meaningful conclusion due to low p-Yki levels. However, since this regulation seems to be conserved in vertebrates, we addressed this question in HEK293T cells. In this experiment, we used cofilin siRNA to downregulate its expression levels. In fact, we observed a reduction in total YAP as well as in p-YAP (indicated by pS127- and pS381-YAP) levels (Fig. S9). These results suggest that both the amount and the phosphorylation status of Yki depend on Tsr.

Taken together, our data suggest that $\mathrm{Tsr}$ is a negative regulator of $\mathrm{Yki}$ nuclear localization and its transcriptional activity.

\section{Yorkie and Twinstar cooperate to coordinate apical membrane expansion}

Increased tube elongation in yki mutants is not caused by an increase in cell number (Fig. S10, A and B), suggesting a different cellular/molecular mechanism, e.g. changes in cell shape. To quantify cell shape, we examined the apical and basal domains of tracheal cells, marked by Uninflatable (Uif) and Perlecan, respectively. In wild-type embryos, the two membrane markers are lined approximately parallel along the tube length (Fig. 8 A-A" and C-C"). In contrast, the DTs of $y k i$ (Fig. 8 B-B") and $t s r^{k 05633}$ (Fig. 8 D-D") mutants showed increased apical membrane with irregular shape, but unaffected basal membrane size, suggesting that only the apical membranes of tracheal cells are over-elongated. Quantification using the adherens junction (AJ) protein Echinoid (Ed) revealed significant increase in the surface of the apical membrane of about $17.2 \%$ in $y k i$ and $18.3 \%$ in $t s r^{k 05633}$ mutants (Fig. 8 F, J, M and $\mathrm{N}$ ), as compared to wild type cells (Fig. 8, E, I, M and N). In line with the 
mutual stabilization of Tsr and Yki at the protein level (Fig. 6, P-R), tracheaespecific expression of $t s r$ or $y k i$ rescued the $y k i$ and $t s r^{k 05633}$ mutant expansion defects, respectively (Fig. 8, G, K, M and N). Based on these experiments, we conclude that increase apical membrane size responsible for changes of tracheal epithelial cells that induce changes in tube size through Tsr/Yki.

\section{Loss of Twinstar and Yorkie affect tube length through changes in F- actin organization}

We next sought to identify players involved in apical membrane mediated changes in tube size. Loss of tsr or $y k i$ (this study) and increased F-actin polymerization (Sansores-Garcia et al., 2011) both cause cell polarityindependent overgrowth. In addition, increased F-actin at the apical surface enhances Yki-mediated gene expression in wing imaginal discs (Fernandez et al., 2011). Moreover, decreased cortical F-actin can lead to increased expansion of the apical cell membrane due to lack of the formation of a contractile network (Forest et al., 2018; Haigo et al., 2003; Kinoshita et al., 2008; Lee and Harland, 2007; Spencer et al., 2015; Tsoumpekos et al., 2018). These observations suggested a link between the loss of $y k i$ and F-actin modulation. Tsr is an actin depolymerization factor that catalyzes F-actin disassembly (Bamburg, 1999). Therefore, we set out to investigate actin distribution in $y k i$ and $t s r^{k 05633}$ mutants. In stage 17 wild type embryos, F-actin (marked by Utrophin and Lifeact) is enriched at the apical cortex of wild type tracheal cells (Fig. 9A, A', C, C'). F-actin accumulates even more in the apical cortex of DT cells of $t s r^{k 05633}$ (Fig. 9B, B') 9 A-B') and yki (Fig. 9, D, D') mutants. To better follow Yki localization, we generated an endogenous 
CRISPR knock-in line expressing an N-terminal tagged form of Yki (called mKate2-Yki). mKate-Yki flies are homozygous viable and fertile, indicating that the generated $y k i$ allele is fully functional, as judged by its ability to rescue a yki null mutation in transheterozygote animals (Fig. S11 $\mathrm{A}$ and $\mathrm{B}$ ).

At 17h AEL (after egg laying), mKate2-Yki starts to accumulate at the apical cortex. This apical enrichment of Yki becomes progressively more prominent at 20h AEL (Fig. $10 \mathrm{~A}$ and Fig. S11 C). This pattern mirrors the endogenous Yki localization, since wild type tracheal tubes of stage 17 embryos, stained with a Yki-specific antibody showed a similar apical enrichment, which was hardly detected in yki mutant embryos (Fig. $10 \mathrm{~B}$ and C). To quantify the amount of Yki in the cortex of tracheal cells in comparison to that in other cellular compartments, such as the nucleus and the residual cytoplasm (Fig. 11 A), we performed Fluorescence Correlation Spectroscopy (FCS) analysis in confocal sections of the cortical cellular domain and in sections of the non-cortical cytoplasm in tracheae of stage 17 embryos (Fig. $11 \mathrm{~B})$. FCS is a well-established method with single-molecule sensitivity to study the dynamic behavior of diffusing fluorescent molecules in live cells or tissues, and extract information about their absolute concentrations and molecular movement (diffusion) within cells or cellular compartments (Vukojevic et al., 2010; Vukojevic et al., 2005). In this way, molecular events, such as binding of protein molecules to larger, immobile structures, resulting in retardation of their diffusion, can be studied with very high sensitivity. A summary on FCS methodology is outlined in Supplement 2. We performed FCS measurements using either a rescuing construct of $y k i$, Yki-GFP (Oh and Irvine, 2008) or the endogenously tagged mKate2-Yki. The concentration of 
Yki-GFP and mKate2-Yki was significantly higher in the apical cortex of wildtype tracheal cells and decreased from the cortex to the cytoplasm to the nucleus (Fig. $11 \mathrm{C}, \mathrm{E}$ and $\mathrm{G}$ ). FCS allowed us to also discern the relative fractions of fast-diffusing versus slowly-diffusing Yki-GFP molecules in these compartments, which indicate the percentage of the total Yki molecules that are presumably docked to larger immobile structures, as compared to those which are freely moving in each of the cellular compartments. A higher fraction of slowly diffusing Yki-GFP and mKate2-Yki molecules was observed in the cortex, whereas less Yki-GFP and mKate2-Yki molecules were found in the cytoplasm and the nucleus (Fig. 11 D, F and H). From this, we conclude that in tracheal cells a higher fraction of $\mathrm{Yki}$ is stabilized at the apical cortex. Based on our results, Tsr is a likely candidate accounting for this stabilization.

Taken together, our results indicate a synergy of Tsr and Yki in the regulation of the actin cytoskeleton to modulate the size of the apical surface of tracheal cells and thereby restrict tube elongation.

\section{Discussion}

Data presented in our study reveal a dual function of Drosophila Yki in tracheal development. First, Yki is required to ensure cuticle water-tightness and second, to restrict tube length.

We show that $y k i$ mutant embryos fail to generate functional gas-filled airways, due to improper establishment of the apical extracellular di-tyrosine network, a structural constituent of the apical ECM, important for tissue integrity. Without excluding additional mechanisms, we have attributed this function to Alas, mutations of which phenocopy the gas-filling defects of $y k i$ 
mutants. We show that Alas is a transcriptional target of $y k i$ and overexpression of Alas rescues the apical intercellular barrier abnormalities and gas-filling defects of $y k i$ mutant embryos, but not the abnormal tube elongation. Therefore, we conclude that $y k i$ regulates tube impermeability through Alas.

Second, results presented here are the first to unveil a molecular mechanism by which Yki restricts tube length in Drosophila airways. We identified Drosophila Tsr/Cofilin as the most abundant Yki interactor in tracheal tissue. Tsr/Cofilin has been shown to bind to both monomeric globular (G)-actin and filamentous (F)-actin, and severs F-actin, thus causing its depolymerization (Andrianantoandro and Pollard, 2006; Bernstein and Bamburg, 2010; Moon and Drubin, 1995). Therefore, regulation of Tsr/Cofilin is critical for adjusting actin dynamics during tissue morphogenesis (Kiuchi et al., 2011). Here, we provide data to suggest that Tsr forms a protein complex with Yki to restrict tracheal tube elongation through regulation of actin polymerization. Depletion of either tsr or $y k i$ induces increased cortical F-actin. As a consequence, the apical membrane expands, and thus tubes grow in length. This finding is in line with studies showing that disassembly of F-actin fosters apical constriction in cells of the early Drosophila embryo (Jodoin et al., 2015). The observed Yki-Tsr interplay is consistent with findings in mammary epithelial cells (MEC) (Aragona et al., 2013), squamous carcinoma cells (SCC) (Kanellos et al., 2015) and in cells of the Drosophila wing epithelium (Ko et al., 2016). Cofilin/Tsr-depletion in these cells induced upregulation of YAP/TAZ/Yki target genes, such as mammalian CTGF (Connective Tissue Growth Factor) or Drosophila Diap1 and expanded 
(Aragona et al., 2013; Ko et al., 2016). Similarly, depletion of capulet, an inhibitor of actin polymerization, or overexpression of a constitutively active allele of diaphanous, encoding a Drosophila formin, which nucleates actin, results in increased F-actin bundles and tissue overgrowth (Sansores-Garcia et al., 2011). However, in other cases, decreased cortical F-actin leads to increased expansion of the apical cell membrane due to lack of the formation of a contractile network (Forest et al., 2018; Haigo et al., 2003; Kinoshita et al., 2008; Lee and Harland, 2007; Spencer et al., 2015; Tsoumpekos et al., 2018), pointing to cell type-specific consequences of apical actin modulation.

How does accumulation of F-actin control Yki activity and thus contribute to organ growth? Several lines of evidence support the notion that mechanical forces, transmitted through the ECM, junctions or the cytoskeleton, act upstream as regulators of Yki in both Drosophila and human cells during development (Dong and Hayashi, 2015; Elbediwy and Thompson, 2018; Schroeder and Halder, 2012; Sun and Irvine, 2016). For example, spectrins, large cytoskeletal proteins and major constituents of the membrane-associated cytocortex (Bennett and Baines, 2001), repress nuclear Yki/YAP activity and therefore tissue growth (Fletcher et al., 2015; Forest et al., 2018). However, our results suggest that the relation between Yki activity and the actin cytoskeleton is not unidirectional and that Yki feeds back to the cytoskeleton via the regulation of Tsr (Choi, 2018).

Yki has been shown to act through thread/Diap1 to restrict tube size (Robbins et al., 2014) and absence of Yki results in reduced transcription of Diap1. While additional mechanistic evidence of the role of Diap1 in actin polymerization and growth control remains to be gathered, earlier studies 
have reported a function of Diap1 in cell migration, independent of its role in apoptosis. The authors showed that border cells of the Drosophila follicle epithelium lacking Diap1 have low levels of F-actin and actin binding proteins and thus fail to migrate (Geisbrecht and Montell, 2004). It will be interesting to explore in future studies whether the defects in tracheal tube length of $y k i$ mutant embryos are due to an additional role of Diap1 in the regulation of the actin cytoskeleton.

Based on our results, we propose a model in which Yki regulates development of the tracheal tubes by at least two mechanisms (Fig. 12). First, apical Yki binds to Tsr and facilitates the docking of a pool of Yki molecules to the apical actin cortex, thereby limiting its nuclear localization, a requirement for transcription of target genes, such as diap1 (Fig. 12A). These findings together with previous observations in wing imaginal disc (Xu et al., 2018) point to a transcription-independent role of Yki in the apical cell cortex to regulate the apical cytoskeleton. Second, residual Yki molecules, which do not bind Tsr, are free to translocate to the nucleus and transcribe Alas to regulate crosslinking of cuticular proteins, and genes involved in in tissue size regulation, such as diap1 (Fig. 12A). Thereby, yki not only regulates tissue size, but also contributes to the establishment of an extracellular barrier necessary for tissue tightness and tracheal gas filling.

Absence of Yki lowers Tsr levels and, therefore results in increased apical F-actin accumulation (Fig. 12B). Furthermore, absence of Yki prevents transcription of Diap1 and Alas, thus giving rise to longer and waterpermeable tubes. The tracheal growth phenotype of yki mutants can be partly be attributed to increased apical F-actin (via reduction of apical Tsr) and partly 
to the absence of Diap1 transcription, since expression of Diap1 in $y k i$ mutants only partially rescues the tube elongation phenotype.

Similarly, in the absence of tsr, the total levels of Yki are decreased but higher amount of Yki molecules translocate to the nucleus to transcribe higher levels of Yki-target genes, e.g. Alas and Diap1 (Fig. 12C). At the same time, apical F-actin accumulates. We, therefore, propose that increased apical Factin, rather than increased expression of Diap1 leads to over-elongation of the tracheal tubes in tsr mutants. Tsr must also be signaling through F-actin to control tube length independently of the Yki/Diap1 pathway. Three lines of evidence favor this hypothesis: i) yki, tsr double mutants show more severe tube length defects as compared to single mutants, indicating that Tsr does not only signal through Yki/Diap1 (Fig. 6 D, E), ii) tracheal expression of $y k i$ in tsr mutants only partially restores the tube length, and iii) tracheal expression of Diap1 partially rescues yki mutants (Fig. 1, E), but not tsr mutants (data not shown).

Similarly, absence of Yki lowers Tsr levels and, therefore results in increased apical F-actin accumulation (Fig. 12C). Furthermore, absence of Yki prevents transcription of Diap1 and Alas, thus giving rise to longer and water-permeable tubes. Thus, the tracheal growth phenotype of $y k i$ mutants can be partly be attributed to increased apical F-actin (via reduction of apical Tsr) and partly to the absence of Diap1 transcription.

Taken together, our data uncover a dual role of $\mathrm{Yki}$ in tracheal development. Yki is required for proper gas-filling and tube growth, two processes that seem to be uncoupled. Overexpression of Alas rescues only the gas filling defects, but not the tube convolution phenotype of $y k i$ mutants. 
Conversely, tracheal length is not altered in Alas mutants. These results provide the first mechanistic view on the role of $\mathrm{Yki}$ in tube length control, which is independent of its role in cell proliferation and apoptosis. Our results contribute to further our understanding of the link between cortical actin organization and apical Yki activity in growth regulation, a link that could also be of importance in the emergence and progression of human diseases.

\section{Materials and Methods}

\section{Fly stocks}

The following Drosophila stocks were used: $w ; y k{ }^{B 5}, F R T 42 D / C y O$ (kindly provided by DJ Pan), UAS-yki.GFP 4-9-Y (3 $3^{\text {rd }}$ chromosome, kindly provided by Kenneth Irvine), w; Mtf ${ }^{\text {ex234 }} / T M 6 C, d f d-Y F P$ (Tiklova et al., 2010), yw; $\quad P\{w[+m C]=l a c W\} D i a p 1[j 5 C 8] / T M 3, S b \quad$ (BDSC \#12093), $\quad y w ;$ $P\{w[+m C]=l a c W\} t s r[k 05633] / C y O \quad$ (BDSC \#12201), yw; $P\{w[+m W . h s]=F R T(w[h s])\} G 13 \operatorname{tsr}[N 96 A] / C y O$ (BDSC \#9108), hpo ${ }^{42-48} / C y O$ (kindly provided by D.J. Pan), $w^{*}$; sp/Cyo; Utr::GFP, Sqh::mCherry/TM3 (kindly provided by Adam Martin), w*; snaSco/CyO; P\{UASt-Lifeact-RFP $\}$ (BDSC \#58362). For rescue experiments, $w ; P\{w[+m C]=U A S-D I A P 1 . H\}$ (BDSC \#6657) w; P\{yw[+mC]=UAS-yki.V5.O\}attP2 (BDSC \#28819), yw; $P\{w[+m C]=U A S-t s r . N\} / T M 6 B, T b \quad(B D S C$ \#9235) were used. Crosses for ectopic expression using bt/-GAL4, w; en-Gal4,UAS.GFP (kindly provided by Georg Halder) were performed at $29^{\circ} \mathrm{C}$. In all experiments $\mathrm{CyO}, \mathrm{TM} 3$, and TM6C balancer strains carrying YFP transgenes were used to identify embryos of the appropriate genotypes. 


\section{Generation of transgenic flies}

To generate the UAS-Alas transgenic line, the alas cDNA (FI09607; obtained from DGRC) was cloned into pJFRC-MUH vector and injected into VK33 fly strain. UAS-Vc.yki (Vc is the C-terminal fragment of GFP) and UAS$\operatorname{Vn} . T s r(\mathrm{Vn}$ is the $\mathrm{N}$-terminal fragment of GFP) were generated by cloning of $y k i$ and tsr cDNA (LD21311 and LD06785 obtained from DGRC) into the pJFRC-MUH vector and injected into VK33 and attp40 fly strains, respectively.

\section{Cas9 genome engineering of $y k i$}

An N-terminal mKate2-Yki knock in chromosome was generated by CRISPR-Cas9 genomic editing using the "Scarless gene editing" design (flycrispr.molbio.wisc.edu), introducing and excisable TTAA-3xP3-dsRedTTAA cassette originally removed by the PiggyBac transposase (Bruckner et al., 2017). The donor vector was modified to be resistant to Cas9 cleavage and bear the mKate 2 and dsRed cassettes. Supplement 3 provides the sequences of the gRNA, its modified version with synonymous mutations in the donor vector and the complete donor vector repair template.

\section{Septate junction permeability assay}

Stage 16.4 embryos (15.5 h AEL) were injected with $10 \mathrm{kDa}$ Rhodamine-Dextran and dye diffusion across epithelia of the main tracheal trunk was monitored by confocal microscopy 1 hour (60 $\mathrm{min}$ ) to 4 hour (240min) after injection. Images were acquired with Zeiss LSM 510 Meta and Zeiss LSM 880 confocal microscopes and processed with Fiji. 


\section{Quantitative Real-Time RT PCR}

Total RNA was extracted from 150 embryos using Ambion RNAqueous kit. cDNA was synthesized using the reverse transcription Master Mix (Applied Biosystems). Real-time PCR was performed using primer sequences of: a) Alas forward primer-ACGGAACGTCTCCTACCTGA b) Alas reverse primerTGCAGGTAGTGTCCGAATTG c) Duox forward primerAGAAAGCAAAAATCGAGTGC d) Duox reverse primerCGGTCTGACTATACATTTTCTCATAA e) tsr forward primerTGTGCGAAATAACCGACCAA f) tsr reverse primerACACCAGAAGCCATTTTTCCT g) yki forward primerGCGCCTTGCCGCCGG h) yki reverse primer-GCTGGCGATATTGGA i) Diap1 forward primer-: TCGTCAAATCTCAAC j) Diap1 reverse primerTGAAGTCGAAACTTG. The experiments were performed for each genotype in triplicate and each experiment was repeated at least three times. Actin probe was used to normalize the total mRNA levels.

\section{Immunohistochemistry}

The following antibodies were used at the indicated dilutions: mouse anti-Coracle (1:100, DSHB), fluorescein-conjugated ChtB (1:500, New England Biolabs), guinea pig anti-Vermiform (1:500; (Tsarouhas et al., 2007)), mouse anti-Crb (Cq4; 1:10, Developmental Studies Hybridoma Bank), mouse anti-Hnt (1:100; Developmental Studies Hybridoma Bank), guinea pig antiYurt (1:1000; gift from Ulrich Tepass), mouse anti-Mega (1:100; gift from Reinhard Schuh), guinea pig anti-Contactin (1:1500; gift from Manzoor Bhat), 
mouse anti-Fasciclin III (1:10; Developmental Studies Hybridoma Bank), rabbit anti-Varicose (1:500; (Bachmann et al., 2008)), guinea pig anti-Gasp (1:800; (Tsarouhas et al., 2007)), rabbit anti-Tsr (1:400; this study), rabbit antiEchinoid (1:1000; gift from Laura Nilson), guinea pig anti-Uif (1:20; gift from Robert Ward), rabbit anti-Perlecan (1:1000; gift from Stefan Baumgartner), anti-Dityrosine (1:200, Japan Institute for the Control of Aging, Cat\# MDT020P), anti-Yki (1:200 for IF, 1:1000 for western blots; gift from Ken Irvine). Wheat germ agglutinin (WGA) (1:200; Thermo Fischer Scientific), chitin binding probe-633 (1:20; gift from Maria Leptin). Secondary antibodies conjugated to Cy3 (Jackson Immunochemicals) or Alexa Fluor 488, 568 and 633 (Molecular Probes) were used at 1:400 dilution. Images were obtained with Zeiss LSM 880 and Zeiss LSM 510Meta confocal setup and processed with Fiji.

\section{Cell number counting}

Stained embryos were imaged with a laser-scanning confocal microscope (LSM780, Carl Zeiss) using a 63/1.4NA C-Apochromat oilimmersion objective. Individual Z stacks were taken with a step size of 0.2-0.5 $\mu \mathrm{m}$ and with a total $\mathrm{Z}$ sampling distance of $20-30 \mu \mathrm{m}$. The cell number counting was based on the nuclei numbers. To separate nuclei closely adjacent to each other (and potentially yielding false negatives), a 3D Watershed segmentation method utilized. Z-stacks binarization, Watershed digital image reconstructions and nuclei counting computed by the ImageJ/Fiji software. 


\section{Proximity ligation assay (PLA)}

PLA recognizes the potential interaction of endogenous proteins using antibodies to detect proteins in close proximity to one another ( $<40 \mathrm{~nm})$. Third instar larva wing discs were dissected and fixed as described above. Primary antibodies against Tsr (rabbit anti-Tsr) and V5 epitope (mouse anti-V5, Invitrogen) were added and incubated overnight at $4^{\circ} \mathrm{C}$. The Duolink PLA Kit (Sigma Aldrich) was used to incubate the tissue with the PLA probes PLUS and MINUS at $37^{\circ} \mathrm{C}$ for 1 hour. Ligation of the PLA oligonucleotides and amplification were performed at $37^{\circ} \mathrm{C}$ for $30 \mathrm{~min}$ and $100 \mathrm{~min}$, respectively. Samples were mounted in Duolink mounting media and imaged using Zeiss LSM 880.

\section{Antibody generation}

Full length tsr of GenBank nucleotide sequence RE04257 was cloned into pGEX4T2 (N-terminus GST) vector using the following primers: forward primer- (BamHI) CTCGGATCCGCTTCTGGTGTAACTGTGTCTG and reverse primer- (EcoRI) CTCGAATTCTTATTGGCGGTCGGTG. The protein was expressed in E.coli BL21 cells, solubilized in $50 \mathrm{mM}$ Tris- $\mathrm{HCl} \mathrm{pH}=8$ and purified using GST Sepharose beads. Purified protein was dialyzed against PBS and used for antibody generation in rabbits at the Charles River Laboratories International, Inc. The rabbit anti-Tsr serum was purified by affinity purification and stored in $50 \%$ glycerol.

\section{Immunoprecipitation}


Fly embryos of the following genotypes: w; btl-Gal4/Cyo,DfdYFP;UASyki.V5 and w; btl-Gal4/Cyo,DfdYFP (as control) were used. Embryos were homogenized on ice using Dounce tissue grinder in $1 \mathrm{~mL}$ of lysis buffer containing $130 \mathrm{mM} \mathrm{NaCl}, 50 \mathrm{mM}$ Tris- $\mathrm{HCL} \mathrm{pH}=8,0,5 \%$ Triton-X and protease inhibitor (Roche). After $30 \mathrm{~min}$ at $4^{\circ} \mathrm{C}$ under rotation the homogenate was centrifuged for $20 \mathrm{~min}$ at $14.000 \mathrm{rpm}$. Supernatant was incubated with the antibody for 2 hours. In the meantime $50 \mu$ l of Protein G were washed 3 times with blocking solution and incubated with the antibody solution overnight at $4^{\circ} \mathrm{C}$. Protein $\mathrm{G}$ beads were collected by centrifugation for 2 min at $3.000 \mathrm{rpm}$ and washed 4 times with lysis buffer. Beads were resuspended in 1.5X SDS sample buffer and heated for $5 \mathrm{~min}$ at $95^{\circ} \mathrm{C}$.

\section{Western Blot}

Samples were separated by SDS-PAGE and blotted onto nitrocellulose 0.45 membrane (Amersham). After blocking in 5\% BSA +TBST, the membrane was incubated overnight with rabbit anti-Tsr diluted 1:4000, antiYki diluted 1:1000 and anti-alpha-Tubulin diluted 1:5000 in blocking buffer. Peroxidase antibodies were used for detection.

\section{Cell Culture and Transfection}

Drosophila S2R ${ }^{+}$cells were cultured at $25^{\circ} \mathrm{C}$ in Schneider's Drosophila medium (Sigma) supplemented with $10 \%$ fetal bovine serum. Transfection of pAct5-Gal4 and Vn-Yki into $\mathrm{S} \mathrm{R}^{+}$cells was performed with FuGENE HD (Promega) according to the manufacturer's protocol. After $48 \mathrm{~h}$, cells were treated with $100 \mu \mathrm{g} / \mathrm{ml}$ cycloheximide for the indicated times. Cells were 
harvested, washed with ice-cold PBS (120 mM NaCl in phosphate buffer at $\mathrm{pH}$ 6.7), resuspended in lysis buffer (containing $10 \%$ glycerol; $1 \%$ Triton $\mathrm{X}$ 100; $1.5 \mathrm{mM} \mathrm{MgCl} ; 120 \mathrm{mM} \mathrm{NaCl} ; 100$ mM PIPES, pH 6.8; 3 mM CaCl $2 ; 1$ $\mathrm{mM}$ PMSF and Complete $\left.{ }^{\mathrm{TM}}\right)$. Cells were lysed on ice for $20 \mathrm{~min}$ and lysates were centrifuged at $14.000 \mathrm{rpm}$ for $20 \mathrm{~min}$ at $4^{\circ} \mathrm{C}$. Sample buffer $3 x$ SDS was added to supernatant and boiled for $5 \mathrm{~min}$ at $95^{\circ} \mathrm{C}$.

\section{Mass spectrometry analysis}

Lysates of stage 17 embryo expressing a tracheae-specific Yki-V5 construct were immunoprecipitated (described above) using the mouse antiV5 antibody (Invitrogen). After electrophoretic separation on SDS gel and Coomassie staining, lanes were cut into 10 slices each, digested in-gel with trypsin, and subjected to GeLC MS/MS analysis.

GeLC MS/MS analysis was performed on an Ultimate3000 nanoLC system interfaced on-line to a LTQ Orbitrap Velos hybrid tandem mass spectrometer (both Thermo Fisher Scientific, Bremen, Germany). Internal standard (GluFib peptide) was spiked into each sample prior analysis. Proteins were identified by Mascot software v.2.2.04 (Matrix Sciences Ltd, London, UK) by searching against Drosophila protein sequences in NCBI database (May 2014, 231613 entries) under the following settings: 5ppm and 0.5Da mass accuracy for precursor and fragment ions, respectively; enzyme specificity - trypsin; maximal number of allowed miscleavages - two; variable modifications - methionine oxidation, N-terminal protein acetylation, cysteine propionamide. The result of the database search was evaluated by Scaffold software v. 4.3.2 (Proteome Software, Portland) using 95\% and 99\% 
probability threshold for peptides and proteins respectively; minimal number of matched peptides was set on two. Calculated False Discovery Rate (FDR) (standards Scaffold feature) for peptides and proteins was below $0.5 \%$. Relative quantification of proteins was performed using MaxQuant software; absolute values of individual proteins were normalized on total protein intensity and internal standard.

\section{Electron microscopy}

Embryos and larvae were fixed in 2\% Glutaraldehyde in $0.1 \mathrm{M}$ PB buffer $\mathrm{pH}=7.2$ for $20 \mathrm{~min}$ at room temperature. Embryos were hand devitellinized. Both embryos and larvae were transferred in microcentrifuge tubes and fixed in $1 \% \mathrm{OsO}_{4} / 2 \%$ Glutaraldehyde and then $2 \% \mathrm{OsO}_{4}$. Specimens were washed and dehydrated in Araldite. Ultrathin sections of $0.1 \mu \mathrm{m}$ were prepared and analyzed with Tecnai 12 BioTWIN (FEI Company).

\section{Fluorescence Microscopy Imaging of live imaginal discs and FCS}

Fluorescence imaging and FCS measurements were performed on two uniquely modified confocal laser scanning microscopy systems, both comprised of the ConfoCor3 system (Carl Zeiss, Jena, Germany) and consisting of either an inverted microscope for transmitted light and epifluorescence (Axiovert $200 \mathrm{M}$ ); a VIS-laser module comprising the Ar/ArKr (458, 477, 488 and $514 \mathrm{~nm}$ ), HeNe $543 \mathrm{~nm}$ and HeNe $633 \mathrm{~nm}$ lasers and the scanning module LSM510 META; or a Zeiss LSM780 inverted setup, comprising Diode 405 nm, Ar multiline 458, 488 and 514 nm, DPSS 561 nm and $\mathrm{HeNe} 633 \mathrm{~nm}$ lasers. Both instruments were modified to enable detection 
using silicon Avalanche Photo Detectors (SPCM-AQR-1X; PerkinElmer, USA) for imaging and FCS. Images were recorded at a 512X512 pixel resolution. CApochromat 40x/1.2 W UV-VIS-IR objectives were used throughout. Fluorescence intensity fluctuations were recorded in arrays of 10 consecutive measurements, each measurement lasting $10 \mathrm{~s}$. Averaged curves were analyzed using the software for online data analysis or exported and fitted offline using the OriginPro 8 data analysis software (OriginLab Corporation, Northampton, MA). In either case, the nonlinear least square fitting of the autocorrelation curve was performed using the Levenberg-Marquardt algorithm. Quality of the fitting was evaluated by visual inspection and by residuals analysis. Control FCS measurements to asses the detection volume were routinely performed prior to data acquisition, using dilute solutions of known concentration of Alexa488 dye. The variability between independent measurements reflects variability between cells, rather than imprecision of FCS measurements. For more details on Fluorescence Microscopy Imaging and FCS, refer to Supplement 2.

\section{Acknowledgments}

We are grateful to Stefan Luschnig, Ulrich Tepass, Reinhard Schuh, Alan Fanning, Manzoor Bhat, Bernard Mechler, Ken Irvine, Stefan Baumgartner, Robert Ward, Maria Leptin, Laura Nilson and Developmental Studies Hybridoma Bank (DSHB; lowa) for sharing antibodies. Adam Martin, Duojia Pan, Ken Irvine, Georg Halder and the Bloomington Drosophila Stock Center for Drosophila strains. The Drosophila Genomics Resource Center (DGRC; 
Indiana) for clones. We are indebted to the Mass Spectrometry Facility and especially to Anna Shevchenko, to the Antibody Facility (Patrick Keller), the Gene Expression Facility (Julia Jarrells), the Light and Electron Microscopy Facility (Jan Peychl and Weihua Leng) at MPI-CBG for the outstanding technical assistance. We also would like to thank Catrin Hälsig, Amelia Aragones-Hernandez and Michaela Burkon for their technical assistance and the Knust lab members for their fruitful discussions. K.S. was supported by a Wenner-Gren stipendium från Wenner-Gren Stiftelserna. The work at MPICBG was supported by the Max-Planck Society.

\section{References}

Andrianantoandro, E., and T.D. Pollard. 2006. Mechanism of actin filament turnover by severing and nucleation at different concentrations of ADF/cofilin. Molecular cell. 24:13-23.

Aragona, M., T. Panciera, A. Manfrin, S. Giulitti, F. Michielin, N. Elvassore, S. Dupont, and S. Piccolo. 2013. A mechanical checkpoint controls multicellular growth through YAP/TAZ regulation by actin-processing factors. Cell. 154:1047-1059.

Bachmann, A., M. Draga, F. Grawe, and E. Knust. 2008. On the role of the MAGUK proteins encoded by Drosophila varicose during embryonic and postembryonic development. BMC developmental biology. 8:55.

Bamburg, J.R. 1999. Proteins of the ADF/cofilin family: essential regulators of actin dynamics. Annual review of cell and developmental biology. 15:185-230.

Beitel, G.J., and M.A. Krasnow. 2000. Genetic control of epithelial tube size in the Drosophila tracheal system. Development. 127:3271-3282.

Bennett, V., and A.J. Baines. 2001. Spectrin and ankyrin-based pathways: metazoan inventions for integrating cells into tissues. Physiological reviews. 81:1353-1392.

Bernstein, B.W., and J.R. Bamburg. 2010. ADF/cofilin: a functional node in cell biology. Trends in cell biology. 20:187-195.

Bruckner, J.J., H. Zhan, S.J. Gratz, M. Rao, F. Ukken, G. Zilberg, and K.M. O'Connor-Giles. 2017. Fife organizes synaptic vesicles and calcium channels for high-probability neurotransmitter release. The Journal of cell biology. 216:231-246. 
Choi, K.W. 2018. Upstream paths for Hippo signaling in Drosophila organ development. BMB reports. 51:134-142.

Dong, B., E. Hannezo, and S. Hayashi. 2014. Balance between apical membrane growth and luminal matrix resistance determines epithelial tubule shape. Cell reports. 7:941-950.

Dong, B., and S. Hayashi. 2015. Shaping of biological tubes by mechanical interaction of cell and extracellular matrix. Current opinion in genetics \& development. 32:129-134.

Edens, W.A., L. Sharling, G. Cheng, R. Shapira, J.M. Kinkade, T. Lee, H.A. Edens, X. Tang, C. Sullards, D.B. Flaherty, G.M. Benian, and J.D. Lambeth. 2001. Tyrosine cross-linking of extracellular matrix is catalyzed by Duox, a multidomain oxidase/peroxidase with homology to the phagocyte oxidase subunit gp91phox. The Journal of cell biology. 154:879-891.

Elbediwy, A., and B.J. Thompson. 2018. Evolution of mechanotransduction via YAP/TAZ in animal epithelia. Current opinion in cell biology. 51:117-123.

Fernandez, B.G., P. Gaspar, C. Bras-Pereira, B. Jezowska, S.R. Rebelo, and F. Janody. 2011. Actin-Capping Protein and the Hippo pathway regulate F-actin and tissue growth in Drosophila. Development. 138:2337-2346.

Fletcher, G.C., A. Elbediwy, I. Khanal, P.S. Ribeiro, N. Tapon, and B.J. Thompson. 2015. The Spectrin cytoskeleton regulates the Hippo signalling pathway. The EMBO journal. 34:940-954.

Forest, E., R. Logeay, C. Geminard, D. Kantar, F. Frayssinoux, L. HeronMilhavet, and A. Djiane. 2018. The apical scaffold big bang binds to spectrins and regulates the growth of Drosophila melanogaster wing discs. The Journal of cell biology. 217:1047-1062.

Forster, D., K. Armbruster, and S. Luschnig. 2010. Sec24-dependent secretion drives cell-autonomous expansion of tracheal tubes in Drosophila. Current biology : CB. 20:62-68.

Forster, D., and S. Luschnig. 2012. Src42A-dependent polarized cell shape changes mediate epithelial tube elongation in Drosophila. Nature cell biology. 14:526-534.

Fulford, A., N. Tapon, and P.S. Ribeiro. 2018. Upstairs, downstairs: spatial regulation of Hippo signalling. Current opinion in cell biology. 51:22-32.

Garud, S.S., and F.F. Willingham. 2012. Molecular analysis of cyst fluid aspiration in the diagnosis and risk assessment of cystic lesions of the pancreas. Clinical and translational science. 5:102-107.

Geisbrecht, E.R., and D.J. Montell. 2004. A role for Drosophila IAP1-mediated caspase inhibition in Rac-dependent cell migration. Cell. 118:111-125.

Haigo, S.L., J.D. Hildebrand, R.M. Harland, and J.B. Wallingford. 2003. Shroom induces apical constriction and is required for hingepoint formation during neural tube closure. Current biology : CB. 13:21252137.

Halder, G., and R.L. Johnson. 2011. Hippo signaling: growth control and beyond. Development. 138:9-22.

Hayashi, S., and B. Dong. 2017. Shape and geometry control of the Drosophila tracheal tubule. Development, growth \& differentiation. 59:4-11. 
Hayashi, S., and T. Kondo. 2018. Development and Function of the Drosophila Tracheal System. Genetics. 209:367-380.

Hu, C.D., Y. Chinenov, and T.K. Kerppola. 2002. Visualization of interactions among bZIP and Rel family proteins in living cells using bimolecular fluorescence complementation. Molecular cell. 9:789-798.

Huang, J., S. Wu, J. Barrera, K. Matthews, and D. Pan. 2005. The Hippo signaling pathway coordinately regulates cell proliferation and apoptosis by inactivating Yorkie, the Drosophila Homolog of YAP. Cell. 122:421-434.

Iruela-Arispe, M.L., and G.J. Beitel. 2013. Tubulogenesis. Development. 140:2851-2855.

Jodoin, J.N., J.S. Coravos, S. Chanet, C.G. Vasquez, M. Tworoger, E.R. Kingston, L.A. Perkins, N. Perrimon, and A.C. Martin. 2015. Stable Force Balance between Epithelial Cells Arises from F-Actin Turnover. Developmental cell. 35:685-697.

Kanellos, G., J. Zhou, H. Patel, R.A. Ridgway, D. Huels, C.B. Gurniak, E. Sandilands, N.O. Carragher, O.J. Sansom, W. Witke, V.G. Brunton, and M.C. Frame. 2015. ADF and Cofilin1 Control Actin Stress Fibers, Nuclear Integrity, and Cell Survival. Cell reports. 13:1949-1964.

Kinoshita, E., E. Kinoshita-Kikuta, K. Takiyama, and T. Koike. 2006. Phosphate-binding tag, a new tool to visualize phosphorylated proteins. Molecular \& cellular proteomics : MCP. 5:749-757.

Kinoshita, N., N. Sasai, K. Misaki, and S. Yonemura. 2008. Apical accumulation of Rho in the neural plate is important for neural plate cell shape change and neural tube formation. Molecular biology of the cell. 19:2289-2299.

Kiuchi, T., T. Nagai, K. Ohashi, and K. Mizuno. 2011. Measurements of spatiotemporal changes in G-actin concentration reveal its effect on stimulus-induced actin assembly and lamellipodium extension. The Journal of cell biology. 193:365-380.

Ko, C., Y.G. Kim, T.P. Le, and K.W. Choi. 2016. Twinstar/cofilin is required for regulation of epithelial integrity and tissue growth in Drosophila. Oncogene. 35:5144-5154.

Laprise, P., S. Beronja, N.F. Silva-Gagliardi, M. Pellikka, A.M. Jensen, C.J. McGlade, and U. Tepass. 2006. The FERM protein Yurt is a negative regulatory component of the Crumbs complex that controls epithelial polarity and apical membrane size. Developmental cell. 11:363-374.

Laprise, P., S.M. Paul, J. Boulanger, R.M. Robbins, G.J. Beitel, and U. Tepass. 2010. Epithelial polarity proteins regulate Drosophila tracheal tube size in parallel to the luminal matrix pathway. Current biology : CB. 20:55-61.

Lee, J.Y., and R.M. Harland. 2007. Actomyosin contractility and microtubules drive apical constriction in Xenopus bottle cells. Developmental biology. 311:40-52.

Llimargas, M., M. Strigini, M. Katidou, D. Karagogeos, and J. Casanova. 2004. Lachesin is a component of a septate junction-based mechanism that controls tube size and epithelial integrity in the Drosophila tracheal system. Development. 131:181-190.

Luschnig, S., T. Batz, K. Armbruster, and M.A. Krasnow. 2006. serpentine and vermiform encode matrix proteins with chitin binding and 
deacetylation domains that limit tracheal tube length in Drosophila. Current biology : CB. 16:186-194.

Moon, A., and D.G. Drubin. 1995. The ADF/cofilin proteins: stimulusresponsive modulators of actin dynamics. Molecular biology of the cell. 6:1423-1431.

Moussian, B. 2010. Recent advances in understanding mechanisms of insect cuticle differentiation. Insect biochemistry and molecular biology. 40:363-375.

Muller, C.B., A. Loman, V. Pacheco, F. Koberling, D. Willbold, W. Richtering, and J. Enderlein. 2008. Precise measurement of diffusion by multicolor dual-focus fluorescence correlation spectroscopy. Epl-Europhys Lett. 83.

Nagaraj, R., S. Gururaja-Rao, K.T. Jones, M. Slattery, N. Negre, D. Braas, H. Christofk, K.P. White, R. Mann, and U. Banerjee. 2012. Control of mitochondrial structure and function by the Yorkie/YAP oncogenic pathway. Genes \& development. 26:2027-2037.

Nelson, K.S., Z. Khan, I. Molnar, J. Mihaly, M. Kaschube, and G.J. Beitel. 2012. Drosophila Src regulates anisotropic apical surface growth to control epithelial tube size. Nature cell biology. 14:518-525.

$\mathrm{Ng}$, J., and L. Luo. 2004. Rho GTPases regulate axon growth through convergent and divergent signaling pathways. Neuron. 44:779-793.

Ochoa-Espinosa, A., M.M. Baer, and M. Affolter. 2012. Tubulogenesis: Src42A goes to great lengths in tube elongation. Current biology : $C B$. 22:R446-449.

Oh, H., and K.D. Irvine. 2008. In vivo regulation of Yorkie phosphorylation and localization. Development. 135:1081-1088.

Oh, H., and K.D. Irvine. 2011. Cooperative regulation of growth by Yorkie and Mad through bantam. Developmental cell. 20:109-122.

Ozturk-Colak, A., B. Moussian, and S.J. Araujo. 2016. Drosophila chitinous $\mathrm{aECM}$ and its cellular interactions during tracheal development. Developmental dynamics : an official publication of the American Association of Anatomists. 245:259-267.

Popoveniuc, G., and J. Jonklaas. 2012. Thyroid nodules. The Medical clinics of North America. 96:329-349.

Rinaldi, P., C. lerardi, M. Costantini, S. Magno, M. Giuliani, P. Belli, and L. Bonomo. 2010. Cystic breast lesions: sonographic findings and clinical management. Journal of ultrasound in medicine : official journal of the American Institute of Ultrasound in Medicine. 29:1617-1626.

Robbins, R.M., S.C. Gbur, and G.J. Beitel. 2014. Non-canonical roles for Yorkie and Drosophila Inhibitor of Apoptosis 1 in epithelial tube size control. PloS one. 9:e101609.

Ryoo, H.D., A. Bergmann, H. Gonen, A. Ciechanover, and H. Steller. 2002. Regulation of Drosophila IAP1 degradation and apoptosis by reaper and ubcD1. Nature cell biology. 4:432-438.

Samakovlis, C., N. Hacohen, G. Manning, D.C. Sutherland, K. Guillemin, and M.A. Krasnow. 1996. Development of the Drosophila tracheal system occurs by a series of morphologically distinct but genetically coupled branching events. Development. 122:1395-1407.

Sansores-Garcia, L., W. Bossuyt, K. Wada, S. Yonemura, C. Tao, H. Sasaki, and G. Halder. 2011. Modulating F-actin organization induces organ 
growth by affecting the Hippo pathway. The EMBO journal. 30:23252335.

Schroeder, M.C., and G. Halder. 2012. Regulation of the Hippo pathway by cell architecture and mechanical signals. Seminars in cell \& developmental biology. 23:803-811.

Shaik, K.S., F. Meyer, A.V. Vazquez, M. Flotenmeyer, M.E. Cerdan, and B. Moussian. 2012. delta-Aminolevulinate synthase is required for apical transcellular barrier formation in the skin of the Drosophila larva. European journal of cell biology. 91:204-215.

Skouloudaki, K., and G. Walz. 2012. YAP1 recruits c-Abl to protect angiomotin-like 1 from Nedd4-mediated degradation. PloS one. 7:e35735.

Soderberg, O., M. Gullberg, M. Jarvius, K. Ridderstrale, K.J. Leuchowius, J. Jarvius, K. Wester, P. Hydbring, F. Bahram, L.G. Larsson, and U. Landegren. 2006. Direct observation of individual endogenous protein complexes in situ by proximity ligation. Nature methods. 3:995-1000.

Spencer, A.K., B.A. Siddiqui, and J.H. Thomas. 2015. Cell shape change and invagination of the cephalic furrow involves reorganization of F-actin. Developmental biology. 402:192-207.

Steinman, T.I. 2012. Polycystic kidney disease: a 2011 update. Current opinion in nephrology and hypertension. 21:189-194.

Sun, S., and K.D. Irvine. 2016. Cellular Organization and Cytoskeletal Regulation of the Hippo Signaling Network. Trends in cell biology. 26:694-704.

Tiklova, K., K.A. Senti, S. Wang, A. Graslund, and C. Samakovlis. 2010. Epithelial septate junction assembly relies on melanotransferrin iron binding and endocytosis in Drosophila. Nature cell biology. 12:10711077.

Tonning, A., J. Hemphala, E. Tang, U. Nannmark, C. Samakovlis, and A. Uv. 2005. A transient luminal chitinous matrix is required to model epithelial tube diameter in the Drosophila trachea. Developmental cell. 9:423430.

Totaro, A., T. Panciera, and S. Piccolo. 2018. YAP/TAZ upstream signals and downstream responses. Nature cell biology. 20:888-899.

Tsarouhas, V., K.A. Senti, S.A. Jayaram, K. Tiklova, J. Hemphala, J. Adler, and C. Samakovlis. 2007. Sequential pulses of apical epithelial secretion and endocytosis drive airway maturation in Drosophila. Developmental cell. 13:214-225.

Tsoumpekos, G., L. Nemetschke, and E. Knust. 2018. Drosophila Big bang regulates the apical cytocortex and wing growth through junctional tension. The Journal of cell biology. 217:1033-1045.

Vukojevic, V., M. Heidkamp, Y. Ming, B. Johansson, L. Terenius, and R. Rigler. 2008. Quantitative single-molecule imaging by confocal laser scanning microscopy. Proc Natl Acad Sci U S A. 105:18176-18181.

Vukojevic, V., D.K. Papadopoulos, L. Terenius, W.J. Gehring, and R. Rigler. 2010. Quantitative study of synthetic Hox transcription factor-DNA interactions in live cells. Proceedings of the National Academy of Sciences of the United States of America. 107:4093-4098.

Vukojevic, V., A. Pramanik, T. Yakovleva, R. Rigler, L. Terenius, and G. Bakalkin. 2005. Study of molecular events in cells by fluorescence 
correlation spectroscopy. Cellular and molecular life sciences : CMLS. 62:535-550.

Wahlstrom, G., M. Vartiainen, L. Yamamoto, P.K. Mattila, P. Lappalainen, and T.I. Heino. 2001. Twinfilin is required for actin-dependent developmental processes in Drosophila. The Journal of cell biology. 155:787-796.

Wang, S., S.A. Jayaram, J. Hemphala, K.A. Senti, V. Tsarouhas, H. Jin, and C. Samakovlis. 2006. Septate-junction-dependent luminal deposition of chitin deacetylases restricts tube elongation in the Drosophila trachea. Current biology : CB. 16:180-185.

Wang, W., J. Huang, and J. Chen. 2011. Angiomotin-like proteins associate with and negatively regulate YAP1. The Journal of biological chemistry. 286:4364-4370.

Wu, V.M., J. Schulte, A. Hirschi, U. Tepass, and G.J. Beitel. 2004. Sinuous is a Drosophila claudin required for septate junction organization and epithelial tube size control. The Journal of cell biology. 164:313-323.

Xu, J., P.J. Vanderzalm, M. Ludwig, T. Su, S.A. Tokamov, and R.G. Fehon. 2018. Yorkie Functions at the Cell Cortex to Promote Myosin Activation in a Non-transcriptional Manner. Developmental cell. 46:271-284 e275.

Yao, L., S. Wang, J.O. Westholm, Q. Dai, R. Matsuda, C. Hosono, S. Bray, E.C. Lai, and C. Samakovlis. 2017. Genome-wide identification of Grainy head targets in Drosophila reveals regulatory interactions with the POU domain transcription factor Vvl. Development. 144:3145-3155.

Zhao, B., L. Li, Q. Lu, L.H. Wang, C.Y. Liu, Q. Lei, and K.L. Guan. 2011. Angiomotin is a novel Hippo pathway component that inhibits YAP oncoprotein. Genes \& development. 25:51-63.

Zuo, L., E. lordanou, R.R. Chandran, and L. Jiang. 2013. Novel mechanisms of tube-size regulation revealed by the Drosophila trachea. Cell and tissue research. 354:343-354. 


\section{Figure Legends}

Figure1. Yki is required to restrict tracheal tube length.

(A) Wild type (WT), $y k i^{B 5}, y k i^{B 5} ; b t />Y k i$ late stage 16 embryos, stained with the chitin binding probe (CBP). Note the convoluted dorsal trunk (DT) tubes in $y k i^{B 5}$ mutant embryos. Tracheae-specific expression of $y k i$ rescues this phenotype. Scale bar: $50 \mu \mathrm{m}$.

(B) DT length is significantly longer in $y k i^{B 5}$ mutants compared to that of wild type (WT) embryos or $y k i^{B 5}$ mutant embryos expressing Yki under the control of the tracheal driver btl-GAL4. Tube length is expressed as a ratio of DT length (metamere 6-10) to body length, normalized against wild type embryos (ratio taken as 1). Included are the measurements of tube length of $m t f$ mutants, a gene encoding a bona fide septate junction component.

(C) Wild type (WT), $y k i^{B 5}, y k i^{B 5} ; b t />Y k i$ late stage 17 embryos. Gas-filling of the DT lumen is observed in wild type and $y k i^{B 5} ; b t />Y k i$, but not in $y k i^{B 5}$ mutant embryos. Scale bar: $20 \mu \mathrm{m}$.

(D) Plot showing the percentage of $y k i^{B 5}$ mutant embryos with gas filling defects, which is significantly different from wild type (WT) and $y k i^{B 5} ; b t />Y k i$ embryos.

(E) The relative tube length of $y k i^{B 5}$ mutant embryos is rescued to different extents by expression of either yki or diap1 by btl-GAL4.

Figure 2. Septate junctions and luminal matrix are not affected in $y k i$ mutant tracheal tubes 
(A-D) Embryonic trachea of wild type (WT) and $y k i^{B 5}$ mutants of stage 17 stained for the core septate junction components, Yurt and Cora (magenta) and the luminal matrix protein Verm (blue). Yurt $(A, C)$ and Cora $(B, D)$ staining appear normal and comparable to that of wild type (WT) embryos. Scale bar: $10 \mu \mathrm{m}$.

(E-G) Transmission electron microscopy of stage 16 embryonic tracheae of wild type (WT) $(E), y k i^{B 5}(F)$ and $m t f(G)$ mutants. Electron dense septa (arrows) are comparable in wild type (WT) and $y k^{i 5}$, but invisible in $m t f$ mutants. Scale bar: $0.5 \mu \mathrm{m}$.

\section{Figure 3. Yki is important for transepithelial barrier function.}

(A-C) Fluorescent 10kDa Dextran injected into the body cavity of embryos at stage 17 does not enter the tracheal lumen (dotted yellow line) of wild type embryos (A). In contrast the dye leaks into the tracheal lumen in $y k i^{B 5}(B)$ mutant embryos, indicating a loss of paracellular barrier. In $y k i^{B 5}$ embryos expressing Yki in the tracheae, the dye is excluded from the lumen, indicating that $y k i$ is required in the tracheae to maintain the barrier function (C). Scale bar: $50 \mu \mathrm{m}$.

(D) Plot representing the percentage of $y k i^{B 5}$, WT and $y k i^{B 5} ; Y k i$ embryos with leakage defects.

(E-G") Time series of Dextran injected embryos. Dextran accumulates gradually in the tracheal lumen of $y k i^{B 5}$ mutant embryos as compared to $m t f$ mutant embryos, where the dye accumulates a few minutes after injection and stays unchanged over time. Scale bar: $100 \mu \mathrm{m}$. 
(H) Quantification of the relative luminal Dextran intensity 60 min (upper plot) and $240 \mathrm{~min}$ (lower plot) after injection.

Figure 4. The apical barrier breaks down in yki mutant embryos.

(A-D) Projections of confocal sections of tracheal dorsal trunks of stage 17 embryos. The di-tyrosine network marking the apical barrier (magenta) is reduced in $A \operatorname{las}^{K G 10015}(A)$ and $y k i^{B 5}(C)$ mutant embryos, whereas the signal is markedly increased in mutant embryos expressing Alas by btl-GAL4 (B, D). White arrows indicate DiTyr apical staining. Scale bar: $20 \mu \mathrm{m}$.

(A'-D') Whole mount embryos at early stage 17. The DT of Alas $^{\text {KG10015 }}{ }^{\left(A^{\prime}\right)}$ and $y k i^{B 5}\left(C^{\prime}\right)$ mutant embryos are not air-filled. In contrast, expression of Alas with the tracheal-specific driver btl-GAL4 rescues the air filling defects of both mutants (B', D'). Scale bar: $20 \mu \mathrm{m}$.

(E) Plot showing the percentage of $A / a s^{K G 10015}$ and $y k^{B 5}$ mutant embryos with gas-filling defects, and significant rescue of this defect in both mutants upon tracheal-specific expression of Alas.

(F) Plot showing the percentage of embryos with defects in the barrier function of the DT, as measured by $10 \mathrm{kDa}$-Rhodamine-dextran leakage into to lumen.

(G) Quantification of anti-Dityrosine intensity as a measure for the apical extracellular barrier.

(H) Quantitative real-time RT-PCR showing a significant difference in Alas mRNA levels between wild type (WT) and $y k i^{B 5}$ mutants at stage 17 . No significant difference was detected in duox mRNA levels. 


\section{Figure 5. Tsr and Yki interact}

(A) Tsr co-immunoprecipitates with Yki from embryo lysates expressing YkiV5 in tracheal cells. bt/-GAL4 alone was used as a negative control. IP, immunoprecipitate; WB, western blot.

(B-C") Proximity ligation assay of Tsr and Yki in wing imaginal discs. Wing discs from en>GFP,Yki-V5 (control) and en>GFP, Yki-V5, Tsr were labeled with anti-V5 and anti-Tsr to perfom PLA assays. The Yki-V5 expression domain is marked by GFP.

(D) Bimolecular Fluorescence Complementation of Yki and Tsr complexes in wing imaginal discs. The diagram depicts the relative BiFC intensity of complexes as compared to control. Scale bars: $50 \mu \mathrm{m}$.

Figure 6. Tsr and Yki act in interconnected pathways to regulate tracheal tube elongation.

(A-E) Loss-of function of tsr (B) causes convoluted DT, similar as loss of $y k i$ (C). This phenotype is enhanced in $t s r^{k 05633} ; y k i^{B 5}$ double mutants (D). (E) Quantification of DT length of wild type (WT), $y k i^{B 5}, t s r^{k 05633}$ and $t s r^{k 05633} ; y k i^{B 5}$ mutants. The DT of $t s r^{k 05633} ; y k i^{B 5}$ double mutants is significantly longer than that of $y k i^{B 5}$ and $t s r^{k 05633}$ single mutants. Scale bar: $50 \mu \mathrm{m}$.

(F-M) Tracheal expression of either $\operatorname{Tsr}(\mathrm{H}, \mathrm{L})$ or $\mathrm{Yki}(\mathrm{I}, \mathrm{M})$ using bt/-GAL4 rescues DT elongation defects of $y k i^{B 5}$ and $t s r^{k 05633}$ mutants. Scale bar: 20 $\mu \mathrm{m}$.

(N) Relative expression of tsr mRNA in wild type (WT), $y k i^{B 5}$ and $t s r^{k 05633}$ mutant embryos of stage 17. tsr mRNA levels are not significantly altered in the absence of $y k i$. 
(O) Relative expression of $y k i$ mRNA in wild type (WT), $y k i^{B 5}$ and $t s r^{k 05633}$ mutant embryos of stage 17. yki mRNA levels are not significantly altered in the absence of $T s r$.

(P) Western blot of protein lysates from wild type (WT), yki ${ }^{B 5}$ and $t s r^{k 05633}$ mutant embryos of stage 17. Note that the protein levels of Tsr and Yki are reduced in the respective other mutant.

$(\mathbf{Q}, \mathbf{R})$ Quantification of the immunoblot in $(P)$ using Fiji, based on the intensity of $\mathrm{Yki}(\mathrm{Q})$ and $\operatorname{Tsr}(\mathrm{R})$ protein, normalized to the loading control (alphaTubulin) ( $n=2)$.

(S) Relative expression of diap1 mRNA in wild type (WT), $y k^{i 55}$ and $t s r^{k 05633}$ mutant embryos of stage 17 . Results were normalized to an endogenous control (actin-5C). Note that diap1 is significantly downregulated in $y k i$ mutants, but significantly upregulated in tsr mutants

Figure 7. Tsr regulates Yki nuclear localization and the expression of its target gene, diap1.

(A,B) Tsr mutant tracheal cells of stage 17 embryos show increased levels of Yki- target gene $\operatorname{diap1-lacZ}(\mathrm{A})$ as compared to the control (B).

Figure 8. Apical membrane expansion contributes to tube overelongation in yki and tsr mutant embryos.

(A-D") Stage 17 wild type (WT) (A-A" and C-C") and $y k i^{B 5}$ mutant (B-B" and D-D") embryos stained with Uif to label the apical membrane (A'-D' and A"-D"' magenta) and Perlecan to label the basement membrane (A-D and A"-D"; green). Scale bars: $20 \mu \mathrm{m}$. 
(E-L) Stage 17 embryos stained with Echinoid to outline the apical surface of DT cells. Scale bars: $10 \mu \mathrm{m}$.

(M) Quantification of the apical surface of $y k i^{B 5}$ and control embryos. A significant increase of the apical surface area is observed in $y k i^{B 5}$ mutants compared to wild type (WT). Apical surface area is restored upon trachealspecific expression of Tsr or Yki in $y k i^{B 5}$ mutant embryos.

(N) Quantification of the apical surface of $t s r^{k 05633}$ and control embryos. A significant increase of the apical surface area is observed in $t s r^{k 05633}$ mutants compared to wild type (WT) embryos. Apical surface area is restored upon tracheal-specific expression of Tsr or Yki in $t s r^{k 05633}$ mutant embryos.

\section{Figure 9. tsr and yki mutant tracheae exhibit increased apical actin}

(A-D') Maximum intensity projections of stage 17 embryos expressing (Utrophin)-GFP (A, B, C, D) and Lifeact-RFP (btl>Lifeact-RFP) (A', B', C', D') to show the apical F-actin cortex in wild type (WT) (A, A', C, C'), $\operatorname{srr}^{k 05633}$ (B, $\left.B^{\prime}\right)$ and $y k i^{B 5}\left(C, C^{\prime}\right)$ mutants. Scale bars: $20 \mu \mathrm{m}$.

Figure 10. Yki is enriched apically in DT cells.

(A) Live imaging of Yki dynamics during late tracheal development, using mKate2-Yki. Apical Yki intensity increases with time. Scale bar: $100 \mu \mathrm{m}$.

(B-C') Confocal images of wild type (WT) (B, B') and $y k i^{B 5}$ mutant (C, C') embryos stained with anti-Yki $(B, C)$ and WGA (green) to label the lumen (dashed lines outline the lumen) (B', $\left.C^{\prime}\right)$. In wild type (WT) embryos, Yki is enriched at the apical cortex (marked by red dashed line) of tracheal cells (B), whereas $y k i^{B 5}$ mutants completely lack Yki cortical labeling $(C)$. (B', C'). Scale 
bar: $10 \mu \mathrm{m}$.

Figure 11. Yki shows the highest concentration at the apical membrane of DT cells.

(A) Cortical and cytoplasmic sections of the tracheal lumen of stage 17 embryos expressing Yki-GFP by btl-Gal4. Scale bar: $5 \mu \mathrm{m}$.

(B) Schematic representation of the different tracheal cell areas, in which the concentration of Yki-GFP was determined by Fluorescence Correlation Spectroscopy (FCS).

(C) Relative Yki-GFP concentrations, as determined by FCS in the cortical and cytoplasmic sections, as well as in the nucleus.

(D) Relative fractions of slowly diffusing Yki-GFP molecules, as measured by FCS. A relatively higher amount of slowly-diffusing Yki-GFP molecules is found in the cortex, as compared to the cytoplasmic area or the nucleus, suggesting more pronounced interactions and binding in the cortex.

(E) Average FCS curves of Yki-GFP in the cortex, cytoplasm and nucleus. The concentration increases from nucleus to cytoplasm to cortex, as shown also in (C).

(F) Normalized average FCS curves to the same amplitude, $G(\tau)=1$, allow comparison of the diffusion of Yki-GFP in the investigated cellular compartments. Yki-GFP displays increasingly slower diffusion from the nucleus to the cytoplasm to the cortex ( $n=36$ cells).

(G) Average FCS curves of mKate2-yki in the cortex, cytoplasm and nucleus.

The concentration increases from nucleus to cytoplasm to cortex. 
(H) Normalized average FCS curves to the same amplitude, $G(\tau)=1$, allow comparison of the diffusion of mKate2-yki in the investigated cellular compartments. mKate2-yki displays increasingly slower diffusion from the nucleus to the cytoplasm to the cortex ( $n=27$ cells).

Figure 12. Model to explain how Yki and Tsr together control tracheal tube growth.

(A) Yki and Tsr cooperate in the apical cell cortex to regulate membrane size and subsequently tissue growth. Tsr is a negative regulator of Yki nuclear translocation. Only a small portion of Yki is able to localize to the nucleus and to transcribe Yki-target genes necessary for tissue growth (e.g. diap1). Yki also transcribes genes required for tissue water-tightness and gas filling (e.g. Alas). Actin is marked in red. Arrow indicates high transcription. Dashed arrow indicates low transcription.

(B) When Yki is absent, Tsr protein levels are reduced, resulting in increased apical F-actin and membrane growth. Yki target genes for tissue growth and water-tightness are no longer transcribed, resulting in longer tubes with defects in gas-filing. Actin is marked in red. Red cross indicates absence of transcription.

(C) In the absence of Tsr, Yki protein levels are reduced and not maintained apically, allowing Yki molecules to translocate to the nucleus, resulting in stronger diap1 transcription. However, higher Diap1 levels do not account for abnormal tube elongation. Rather, F-actin accumulates apically and apical membrane growth is increased, leading in longer tubes. Actin is marked in red. 


\section{Supporting information}

Suppl. Fig 1. Localization of septate junction proteins is not compromised in the absence of Yorkie.

Single confocal sections of early stage 17 dorsal trunks stained for septate junction (SJ) proteins NrxIV, FasIII, Mega, Dlg and Cont. In $y k i^{B 5}$ mutant embryos SJ proteins do not change their expression or localization. Scale bar: $10 \mu \mathrm{m}$.

Suppl. Fig 2. Localization of the luminal matrix protein Serp is not compromised in the absence of Yorkie.

(A-B") Single confocal sections of early stage 17 dorsal trunks stained for the septate junction protein Mtf (A, B and A". B") and the luminal matrix marker, Serp (A', B' and A". B'). In $y k i^{B 5}$ mutant embryos Serp does not change its expression or its localization. Scale bar: $20 \mu \mathrm{m}$.

Suppl. Fig 3. Yorkie levels influence Dityrosine network in wing discs. (A-C") Yki overexpression (A-A") or knockdown (B-B") by ptc-Gal4 leads to increased or decreased levels of dityrosine staining in the A/P boundary of third instar wing discs, respectively. GFP (ptc>GFP) was used as a negative control (C-C"). Scale bar: $100 \mu \mathrm{m}$.

(D) Quantification of relative intensities of dityrosine in the ptc-Gal4 domain.

Suppl. Fig 4. Loss of Alas does not affect axial tube elongation. 
(A-B') Lateral views of $\operatorname{Alas}^{K G 10015}(\mathrm{~A})$ and $y k^{i 5}\left(\mathrm{~A}^{\prime}\right)$ embryos stained with WGA, and of live embryos (B, B') showing that tubes in alas ${ }^{\text {KG10015 }}$ mutants are shorter than in embryos mutants for yki Scale bars: $20 \mu \mathrm{m}\left(A, A^{\prime}, B, B^{\prime}\right)$.

(C) Bar graph showing the relative tube length of $A / a s^{K G 10015}$ and $y k i^{B 5}$ embryos.

Suppl. Fig 5. Cell polarity is preserved in the absence of Yorkie.

(A-B) Confocal projections of the dorsal trunk metamere 8 of early stage 17 wild type (WT) and $y k i^{B 5}$ embryos labeled for Crb.

(A'-B') Superficial sections of the dorsal trunk metamere 8 of early stage 17 wild type and $y k i^{B 5}$ embryos labeled for aPKC. Scale bars: $20 \mu \mathrm{m}$.

Suppl. Fig 6. Twinstar and actin specifically associate with Yorkie.

(A) Mass spectrometric identification and relative quantification of Twinstar protein in repetitive immunoprecipitations of V5-tagged Yorkie.

a - as reported by Scaffold software under setting described in materials and methods section;

b - calculated as ratio between relative normalized intensities in immunoprecipitation and corresponding control; Twinstar was not detected in the control of the experiment $\mathrm{N} 1$.

C - immunoprecipitation of V5-tagged Yorkie and control experiments were performed as described in materials and methods section.

Suppl. Fig 7. Twinstar mutants exhibit defects in tube size, but not gasfilling and paracellular barrier defects. 
(A-C) Tracheal phenotypes of two different alleles of twinstar, $t s r^{k 05633}$ and $t s r^{N 96 A}$, stained for Verm.

(D-E) Overviews of $t s r^{k 05633}$ stage 17 embryos, showing proper tracheal gas filling in the absence (D) or presence (E) of a transgene expressing a wild type Twinstar. Scale bars: $50 \mu m(A, B)$.

(F-G) Fluorescent 10kDa Dextran injected into the body cavity of WT (F) and $t s r^{k 05633}(\mathrm{G})$ mutants does not leak into the tracheal lumen (dashed lines), indicating that the paracellular barrier in $t s r^{k 05633}$ mutants is intact. Scale bar: $50 \mu \mathrm{m}$.

\section{Suppl. Fig 8. Yki and Tsr are maternally contributed}

(A) Protein lysates from unfertilized eggs of $y k^{i 5}$ and $t s r^{k 05633}$ heterozygote females.

(B) Western blots from cell lysates of S2 cells expressing Yki and Tsr, treated with cycloheximide $(\mathrm{CHX})$ for $0,1,2 \ldots .6$ hours. Tubulin is used as a loading control.

(C) Degradation kinetics $\left(N(t)=N_{0} e^{-\lambda t}\right)$, derived from panel $\mathrm{B}$, shows the level of $\mathrm{Vn}-\mathrm{Yki}$ in $\mathrm{S} 2$ cells after protein synthesis inhibition by cycloheximide $(\mathrm{CHX}) .0,1,2 \ldots 18$ refer to hours after $\mathrm{CHX}$ addition. Data were collected from two independent experiments.

Suppl. Fig 9. Knock-down of Cofilin reduces YAP protein levels and decreases its phosphorylation state. 
Cofilin knockdown in HEK293T cells expressing GFP-Cofilin reduces YAP total protein levels as well as YAP phosphorylation at S381 and S127. Tubulin is used as loading control.

\section{Suppl. Fig 10. Tracheal cell number does not change in $y k i^{B 5}$ mutant embryos.}

(A) Confocal projections showing the dorsal trunk (DT) of stage 17 wild type (WT) and $y k i^{B 5}$ mutant embryos stained for the luminal protein Gasp (green) and the nuclear marker Hnt (Hindsight, magenta). Scale bars: 10 $\mathrm{mm}$.

(B) Plot showing the average number of cells, which correspond to the number of marked nuclei, in wild type (WT) $(n=6)$ and $y k i^{B 5}(n=8)$ mutant embryos of the tracheal metamere 6 ( $\operatorname{Tr} 6)$ of stage 17 embryos. Values were calculated based on a 3D Watershed-based image transformation (see details in Materials and Methods). Unpaired two tailed $t$-test was performed to obtain the indicated $p$ value. Error bars represents s.e.m.

Suppl. Fig 11. The endogenously tagged yki allele (mKate2-yki) is homozygous viable and rescues the mutation.

$(\mathrm{A}, \mathrm{B})$ Lateral views of an mKate2-yki $/ y k i^{B 5}(\mathrm{~A})$ transheterozygous and an mKate2-yki homozygous (B) stage 17 embryo showing normal tube length and gas-filling. Scale bar: $20 \mu \mathrm{m}$.

(C) Confocal section of part of the DT of an mKate2-yki embryo of stage 17 expressing Histone-GFP (green). Yki (magenta) is localized apically in the tracheae, but cannot be detected in the nuclei (marked by GFP). Scale bar: 50 $\mu \mathrm{m}$ 


\section{Supplement 1. Overview of actin peptides detected by mass spectrometry in immunoprecipitations of V5-tagged Yorkie and the corresponding controls.}

Peptides detected with mass spectrometry are highlighted with different colors: grey indicates peptides shared between all actin sequences (peptides in italic were used for relative quantification of total actin); yellow - peptides unique for Act5C/Act42A, blue - peptides unique for Act79B/Act88F/Act87E, green - peptides unique for Act57B.

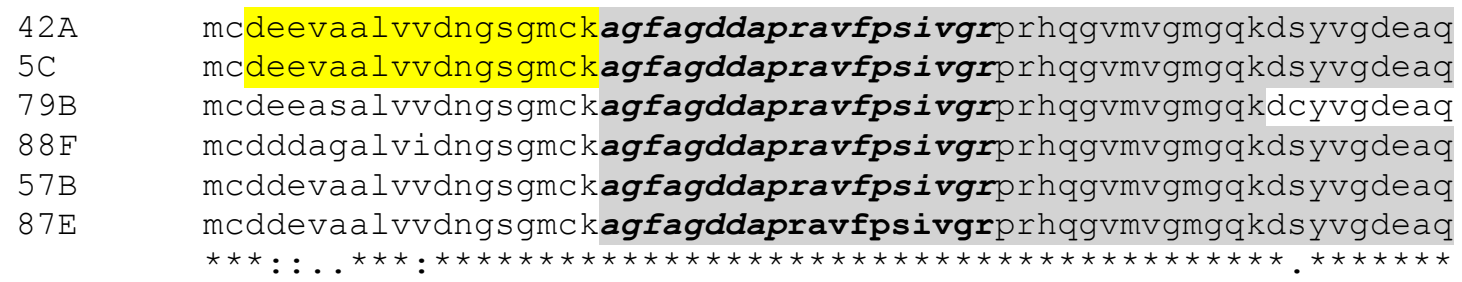

skrgiltlkypiehgivtnwddmekiwhht fynelrvapeehpvll teaplnpkanrekm skrgiltlkypiehgivtnwddmekiwhhtfynelrvapeehpvll teaplnpkanrekm skrgilslkypiehgiitnwddmekvwhht fynelrvapeehpvll teaplnpkanrekm skrgiltlkypiehgiitnwddmekiwhhtfynelrvapeehpvll teaplnpkanrekm skrgiltlkypiehgiitnwddmekiwhht fynelrvapeehpvll teaplnpkanrekm skrgiltlkypiehgiitnwddmekiwhht fynelrvapeehpvll teaplnpkanrekm

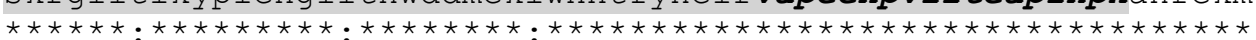
tqimfetfnspamyvaiqavlslyasgrttgivldsgdgvshtvpiyegyalphailrld tqimfet fnspamyvaiqavlslyasgrttgivldsgdgvshtvpiyegfalphailrld tqimfetfnspamyvaiqavlslyasgrttgivldsgdgvshtvpiyegyalphailrld tqimfetfnapamyvaiqavlslyasgrttgivldsgdgvshtvpiyegyalphailrld lagrdltdylmkiltergysfttaereivrdikeklcyvaldfeqemataaastsleks lagrdltdylmkiltergysfttaereivrdikeklcyvaldfeqemataaastsleks lagrdltdylmkiltergysfttaereivrdikeklcyvaldfeqemataaastsleks lagrdltdylmkiltergysfttaereivrdikeklcyvaldfeqemataaastsleks

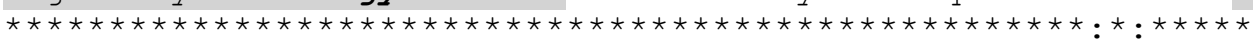


Supplement 2. Background on Fluorescence Microscopy Imaging and FCS.

Two individually modified instruments (Zeiss, LSM 510 and 780, ConfoCor 3) with fully integrated FCS/CLSM optical pathways were used for imaging. The detection efficiency of CLSM imaging was significantly improved by the introduction of APD detectors. As compared to PMTs, which are normally used as detectors in conventional CLSM, the APDs are characterized by higher quantum yield and collection efficiency - about $70 \%$ in APDs as compared to $15-25 \%$ in PMTs, higher gain, negligible dark current and better efficiency in the red part of the spectrum. Enhanced fluorescence detection efficiency enabled image collection using fast scanning ( 1 $5 \mu \mathrm{s} /$ pixel ). This enhances further the signal-to-noise-ratio by avoiding fluorescence loss due to triplet state formation, enabling fluorescence imaging with single-molecule sensitivity. In addition, low laser intensities (150-750 $\mu W)$ could be applied for imaging, significantly reducing the phototoxicity (Vukojevic et al., 2008).

FCS measurements are performed by recording fluorescence intensity fluctuations in a very small, approximately ellipsoidal observation volume 
element (OVE) (about $0.2 \mu \mathrm{m}$ wide and $1 \mu \mathrm{m}$ long) that is generated in trachea epithelial cells by focusing the laser light through the microscope objective and by collecting the fluorescence light through the same objective using a pinhole in front of the detector to block out-of-focus light. The fluorescence intensity fluctuations, caused by fluorescently labeled molecules passing through the OVE are analyzed using temporal autocorrelation analysis.

In temporal autocorrelation analysis we first derive the autocorrelation function $G(\tau)$ :

$$
G(\tau)=1+\frac{\langle\delta I(t) \cdot \delta I(t+\tau)\rangle}{\langle I(t)\rangle^{2}}(\mathrm{~S} 1)
$$

where $\delta I(t)=I(t)-\langle I(t)\rangle$ is the deviation from the mean intensity at time $t$ and $\delta I(t+\tau)=I(t+\tau)-\langle I(t)\rangle$ is the deviation from the mean intensity at time $t+\tau$. For further analysis, an autocorrelation curve is derived by plotting $G(\tau)$ as a function of the lag time, i.e. the autocorrelation time $\tau$.

To derive information about molecular numbers and their corresponding diffusion time, the experimentally obtained autocorrelation curves are compared to autocorrelation functions derived for different model systems. A model describing free three dimensional (3D) diffusion of two components and triplet formation was used in this study:

$$
\begin{gathered}
G(\tau)=1+\frac{1}{N}\left(\frac{1-y}{\left(1+\frac{\tau}{\tau_{D_{1}}}\right) \cdot \sqrt{1+\frac{w_{x y}^{2} \tau}{w_{z}^{2} \tau_{D_{1}}}}}+\frac{y}{\left(1+\frac{\tau}{\tau_{D_{2}}}\right) \cdot \sqrt{1+\frac{w_{x y}^{2} \tau}{w_{z}^{2} \tau_{D_{2}}}}}\right) \\
\cdot\left(1+\frac{T}{1-T} e^{-\frac{\tau}{\tau_{T}}}\right)(\mathrm{S} 2)
\end{gathered}
$$


In the above equation, $N$ is the average number of molecules in the OVE; $y$ is the fraction of the slowly moving Yki-GFP molecules; $\tau_{D_{1}}$ is the diffusion time of the free Yki-GFP molecules; $\tau_{D_{2}}$ is the diffusion time of Yki-GFP molecules undergoing interactions with the DNA; $w_{x y}$ and $w_{z}$ are radial and axial parameters, respectively, related to spatial properties of the OVE; $T$ is the average equilibrium fraction of molecules in the triplet state; and $\tau_{T}$ the triplet correlation time related to rate constants for intersystem crossing and the triplet decay. Spatial properties of the detection volume, represented by the square of the ratio of the axial and radial parameters $\left(\left(\frac{w_{z}}{w_{x y}}\right)^{2}\right)$, are determined in calibration measurements performed using a solution of Rhodamine 6G for which the diffusion coefficient (D) is known to be $D_{R h 6 G}=4.1 \cdot 10^{-10} \mathrm{~m}^{2} \mathrm{~s}^{-1}$ (Muller et al., 2008). The diffusion time, $\tau_{D}$, measured by FCS, is related to the translation diffusion coefficient $D$ by:

$$
\tau_{D}=\frac{w_{x y}^{2}}{4 D}(\mathrm{~S} 3)
$$

To establish that Yki molecules diffusing through the OVE are the underlying cause of the recorded fluorescence intensity fluctuations, we plotted the characteristic decay times $\tau_{D 1}$ and $\tau_{D 2}$, obtained by FCS, as a function of the total concentration of Yki molecules. We observed that both characteristic decay times remain stable for increasing total concentration of Yki molecules, signifying that the underlying process triggering the fluorescence intensity fluctuations is diffusion of fluorescent Yki molecules through the OVE (which should be independent of the total concentration of Yki molecules). 


\section{Supplement 3. Sequences for the generation of the CRISPR knock-in} line expressing an N-terminal tagged form of Yki (called mKate2-Yki).

The following sequences were used:

a) yki gRNA: 5'-ccttcttcacgeccccggcgecc-3'

b) yki gRNA modified sequence in the donor vector: 5'cgtttttacccogccogcccog-3'

c) donor vector for Yki Knockin with dsRed excisable cassette: cccagcccgcctaatgagcgggctttttttgaacaaaacaacttatatcgtatggggctgacttcaggtgcta catttgaagagataaattgcactgaaatctagaaatattttatctgattaataagatgatcttcttgagatcgttttg gtctgcgcgtaatctcttgctctgaaaacgaaaaaaccgccttgcagggcggtttttcgaaggttctctgagct accaactctttgaaccgaggtaactggettggaggagcgcagtcaccaaaacttgtcctttcagtttagcctta accggcgcatgacttcaagactaactcctctaaatcaattaccagtggctgctgccagtggtgcttttgcatgt ctttccgggttggactcaagacgatagttaccggataaggcgcagcggtcggactgaacggggggttcgtg catacagtccagcttggagcgaactgcctacccggaactgagtgtcaggcgtggaatgagacaaacgcg gccataacagcggaatgacaccggtaaaccgaaaggcaggaacaggagagcgcacgagggagccg ccagggggaaacgcctggtatctttatagtcctgtcgggtttcgccaccactgatttgagcgtcagatttcgtga tgcttgtcagggggggggagcctatggaaaaacggetttgccgcggccctctcacttccctgttaagtatcttc ctggcatcttccaggaaatctccgccccgttcgtaagccatttccgctcgccgcagtcgaacgaccgagcgt agcgagtcagtgagcgaggaagcggaatatatcctgtatcacatattctgctgacgcaccggtgcagccttt tttctcctgccacatgaagcacttcactgacaccctcatcagtgccaacatagtaagccagtatacactccgc tagcgcgaggagcgcagtcacctctagatgtgacggaagatcacttcgcagaataaataaatcctggtgtc cctgttgataccgggaagcctgggccaactttggcgaaaatgagacgttgatcggcacgtaagaggttc caactttcaccataatgaaataagatcactaccgggcgtatttttgagttatcgagattttcaggagctaagga agctaaaatggagaaaaaaatcactggatataccaccgttgatatatcccaatggcatcgtaaagaacattt tgaggcatttcagtcagttgctcaatgtacctataaccagaccgttcagctggatattacggccttttaaagac 
cgtaaagaaaaataagcacaagttttatccggcctttattcacattcttgcccgcctgatgaatgctcatccgg aattccgtatggcaatgaaagacggtgagctggtgatatgggatagtgttcaccettgttacaccgttttccatg agcaaactgaaacgttttcatcgctctggagtgaataccacgacgatttccggcagtttctacacatatattcg caagatgtggcgtgttacggtgaaaacctggcctatttccctaaagggtttattgagaatatgttttcgtctcag ccaatccctgggtgagtttcaccagtttgatttaaacgtggccaatatggacaacttcttcgcccccgttttcac catgggcaaatattatacgcaaggcgacaaggtgctgatgccgctggcgattcaggttcatcatgccgtctg tgatggcttccatgtcggcagaatgcttaatgaattacaacagtactgcgatgagtggcagggcggggcgta accatggatccatggttagccctccacacataaccaggaggtcagattggtaccaatcggtgcttactcat ctgaggaacgaaagcaagcaatatttacctcgaatatccttaggaggaggttcacgtacgcttttcggacgc cgattgatttgtttatggcggaaataaatatgagaaatgagaggaagcccccgatggggatccagaatatgt ccaacagcacggcgatcattttacatttgtgtcatgcgacattagttattgttaagcgaaaaatgcctccgaatt gtgtcgtgccaatatatacaatgttaaaatacttttgctgtagaccgaactagcgatggaccttgactgtgtca aaaacctgtctcgtgctcacagacctggcccgcgctcatctctacggggaacaaacggaaaggggacga ttccggtcgggcaggagacggaacggcttcttgttccactgccaatcgtttgaaaagggttgaatgaacgtg cactgaaaacaaaaaggcacatttacattcagattatattgttattgggtatgtgtttttgacagacctttacctgc acttattacttaaatcaacaaggcacatttacaatcagtttatattgttattgggtgtgttttgacagacctttgcct gtacttattttcactaaacaaaatgttataaaccaataaataataattgttagtctaataatttatagtctgatattat ggaacacaagtgtgtgggctatacacacaccataatttaatatctactttgtttgtgccttattaattacaaaata tagaataaatctttagctatagctaaaggaaatcgacaaaagtcgtaatcggcttgcggaataccctggca ttccgcaggtgcagccacgaagccgattacatgaggaacgctagtatcggaatggacaagtggacgggg atagccatctggcaacactgggataaatttattttatgttggcagttccgtaattattattattactattatttattgca acgaagtttagttttaaccctagaaagataatcatattgtgacgtacgttaaagataatcatgcgtaaaattga cgcatgtgttttatcggtctgtatatcgaggtttatttattaatttgaatagatattaagttttattatatttacacttacat actaataataaattcaacaaacaatttatttatgtttatttatttattaaaaaaaaacaaaaactcaaaatttcttct ataaagtaacaaaacttttaggatctaattcaattagagactaattcaattagagctaattcaattaggatcca 
agcttatcgatttcgaaccctcgaccgccggagtataaatagaggcgettcgtctacggagcgacaattcaa ttcaaacaagcaaagtgaacacgtcgctaagcgaaagctaagcaaataaacaagcgcagctgaacaa gctaaacaatcggctcgaagccggtcgccaccatggcctcctccgaggacgtcatcaaggagttcatgcg cttcaaggtgcgcatggagggctccgtgaacggccacgagttcgagatcgagggcgagggcgagggec gccctacgagggcacccagaccgccaagctgaaggtgaccaagggcggccccctgccettcgcctgg gacatcctgtcccccagttccagtacggetccaaggtgtacgtgaagcaccccgccgacatccccgacta caagaagctgtccttccccgagggcttcaagtgggagcgcgtgatgaacttcgaggacggcggcgtggtg accgtgacccaggactcctccctccaggacggetccttcatctacaaggtgaagttcatcggcgtgaacttc ccctccgacggecccgtaatgcagaagaagactatgggetgggaggcgtccaccgagcgcctgtacccc cgcgacggcgtgctgaagggcgagatccacaaggccetgaagctgaaggacggcggccactacctggt ggagttcaagtccatctacatggccaagaagcccgtgcagetgcccggctactactacgtggactccaagc tggacatcacctccacaacgaggactacaccatcgtggagcagtacgagcgcgccgagggccgccac cacctgttcctgtagcggccgcgactctagatcataatcagccataccacatttgtagaggtttacttgctttaa aaaacctcccacacctcccctgaacctgaaacataaaatgaatgcaattgttgttgttaacttgtttattgcag cttataatggttacaaataaagcaatagcatcacaaatttcacaaataaagcattttttcactgcattctagttgt ggtttgtccaaactcatcaatgtatcttagatatctataacaagaaaatatatatataataagttatcacgtaagt agaacatgaaataacaatataattatcgtatgagttaaatcttaaaagtcacgtaaaagataatcatgcgtca tttgactcacgcggtcgttatagttcaaaatcagtgacacttaccgcattgacaagcacgcctcacgggagc tccaagcggcgactgagatgtcctaaatgcacagcgacggattcgcgctatttagaaagagagagcaata tttcaagaatgcatgcgtcaattttacgcagactatctttctagggttaacaccttaatgttatagtttcgcgcagc gcgatggagtgatacgtatatggaagaacatatatgtgcgcgtgcctaatcgctaagataattctatgtagtttt cgtttgtatacaataagtgccttttatatggtgagcgagctgattaaggagaacatgcacatgaagctgtacat ggagggcaccgtgaacaaccaccacttcaagtgcacatccgagggcgaaggcaagcctacgagggc acccagaccatgagaatcaaggcggtcgagggeggecctctcccttcgecttcgacatcctggetacca gcttcatgtacggcagcaaaaccttcatcaaccacacccagggcatccccgacttctttaagcagtccttccc 
cgagggcttcacatgggagagagtcaccacatacgaagacgggggcgtgctgaccgctacccaggaca ccagcctccaggacggctgcctcatctacaacgtcaagatcagaggggtgaacttcccatccaacggecc tgtgatgcagaagaaaacactcggctgggaggcctccaccgagaccctgtacccogctgacggcggcct ggaaggcagagccgacatggccctgaagctcgtgggcgggggccacctgatctgcaacttgaagacca catacagatccaagaaacccgctaagaacctcaagatgcccggcgtctactatgtggacagaagactgg aaagaatcaaggaggccgacaaagagacctacgtcgagcagcacgaggtggctgtggccagatactg cgacctccctagcaaactggggcacagaggtggaggaggtatgttaacgacgatgtcagccagcagca atacaaacagcctgatcgagaaggagatcgacgacgaggacatgctttcgccgatcaagtccaacaacc tggtggtgcgggtcaaccaggacacggacgacaacctgcaggcgctattcgacagcgtcctgaatccgg gtgacgccaagcgcccgctgcagctgcccctgcgcatgcggaagctgcccaactcgtttttaccccgccc gccccgtcgcactcgcgggccaacagcgccgactccacctacgacgcgggctcccagtcgagcatcaa catcgggaacaaggcgtccatcgtccagcagccagatggccagtcgcccatcgccgccatcccccagct ccagattcagccgtctccccagcacagccgcctggcgatacatcactcccgagcccgcagcagccccgc ctcgctgcagcagaactacaatgtgcgcgcccggagcgacgcagcagcagccaacaatccgaatgcca atccgagcagccaacagcagcccgctgggcccactttcccagagaacagtgcccaagagttccccagc ggcgcccoggccagctcggccattgatctggacgccatgaacacctgcatgtcgcaggacattcccatgtc catgcagacagtgcacaagaagcagcgctcctacgacgtcatcagccccattcagttgaaccgccaacta ggcgccttgccgccgggatgggagcaagccaagaccaatgatggccagatctactacttgaagtgagttt gggcagattttaaaaatgatatgcatgaagctttatttaatacgtgtcttctttatgaacttatgaaaactaaaata ttcttgtgagtcacagaaatctcattggcttcgttggatgtctttcaagtttatctcttaatcattactagctcaacta atacccaaaacagcaatctcaacaatggtgttctctctacttgcagtcatactacaaaatctacgcagtggga ggatcccagaatccaatatcgccagcagcagcaaatcttgatggccgagcgaataaagcagaatggtag gttctatcttagatgtaatgccttagataagcaagtcgttaactgcctgtggctgcgtctcattgttaacccactta aatcagccatcagaggactaattaatgctagagaccaggcagggataagataggctctaactcattccaa ctaacgcaacgcgtgctagcgctttgtttattttctaaatacattcaaatatgtatccgctcatgagacaataac 
cctgataaatgcttcaataatattgaaaaaggaagagtatgagtattcaacatttccgtgtcgcccttattccctt tttgcggcattttgccttcctgtttttgctcacccagaaacgctggtgaaagtaaaagatgctgaagatcagttg ggtgcacgagtgggttacatcgaactggatctcaacagcggtaagatccttgagagttttcgccccgaaga acgtttccaatgatgagcacttttaaagttctgctatgtggcgcggtattatcccgtgttgacgccgggcaaga gcaactcggtcgccgcatacactattctcagaatgacttggttgagtactcaccagtcacagaaaagcatctt acggatggcatgacagtaagagaattatgcagtgctgccataaccatgagtgataacactgcggccaactt acttctgacaacgatcggaggaccgaaggagctaaccgctttttgcacaacatgggggatcatgtaactc gccttgatcgttgggaaccggagctgaatgaagccataccaaacgacgagcgtgacaccacgatgcctg cagcaatggcaacaacgttgcgcaaactattaactggcgaactacttactctagcttcccggcaacaattaa tagactggatggaggcggataaagttgcaggaccacttctgcgctcggccettccggctggctggtttattgct gataaatctggagccggtgagcgtgggtctcgcggtatcattgcagcactggggccagatggtaagccctc ccgtatcgtagttatctacacgacggggagtcaggcaactatggatgaacgaaatagacagatcgctgag ataggtgcctcactgattaagcattggtaa 
A
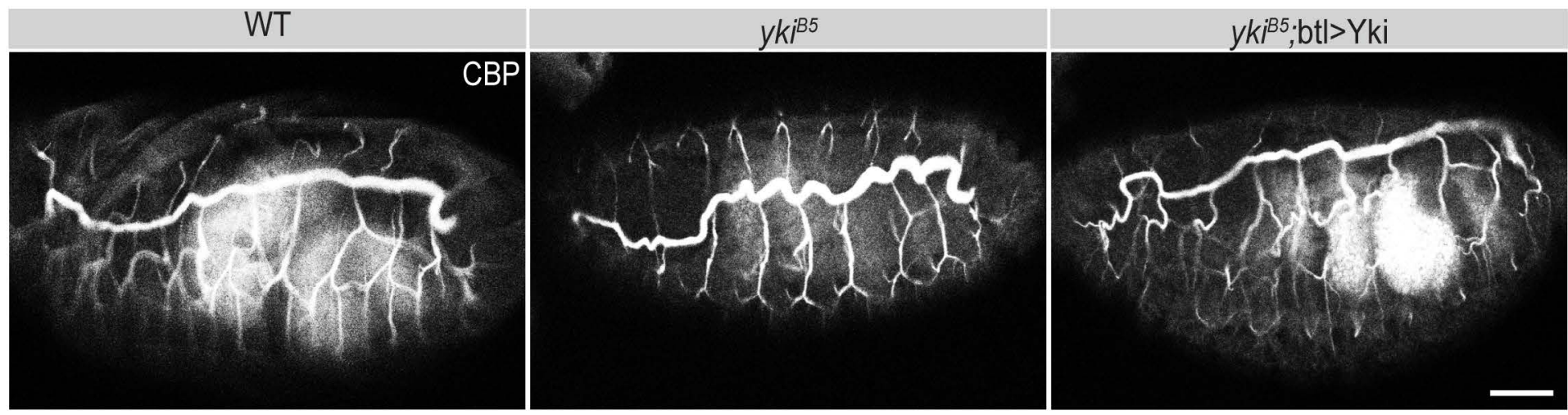

B
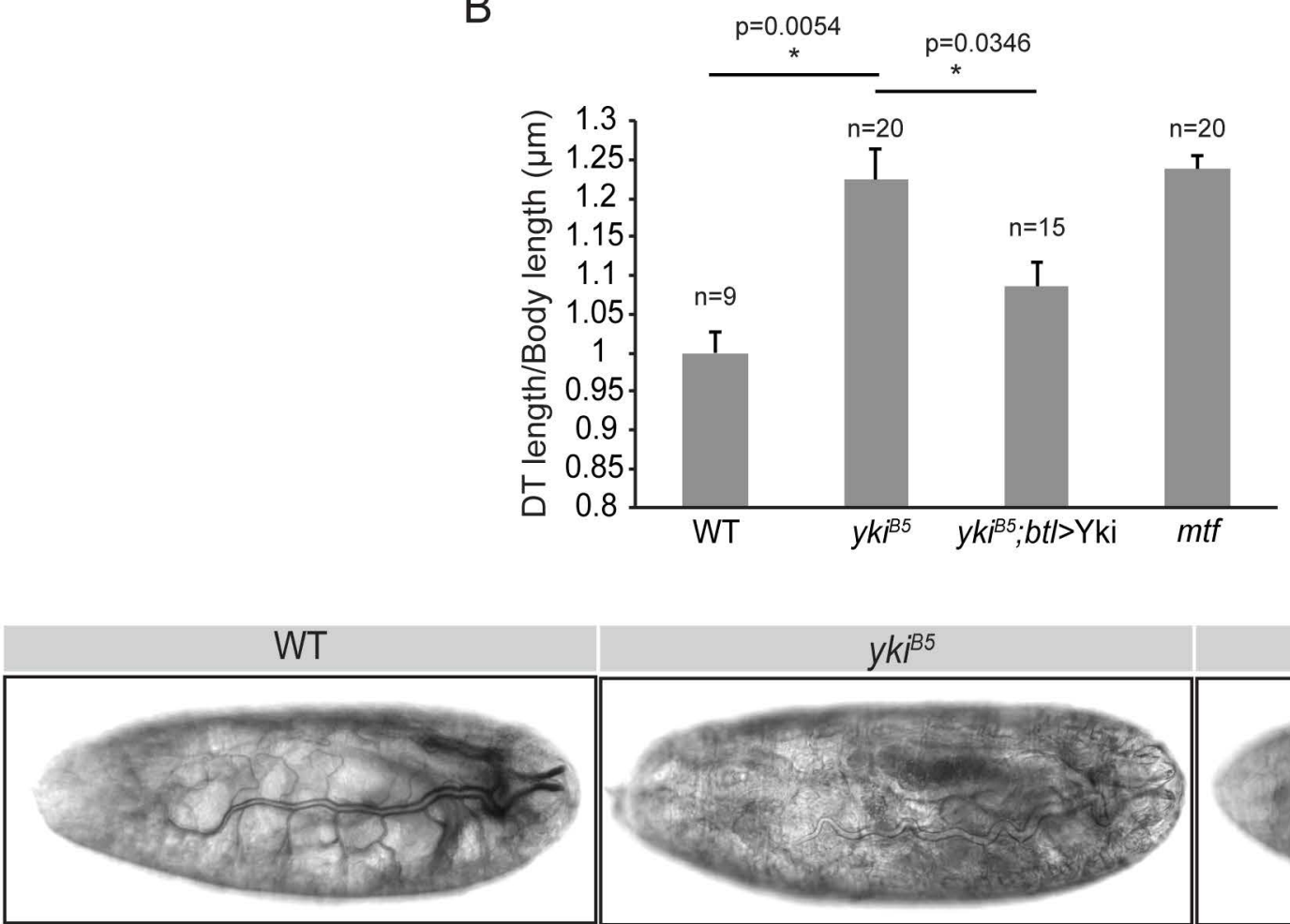

$y_{k j}{ }^{i 5}$;btl>Yki
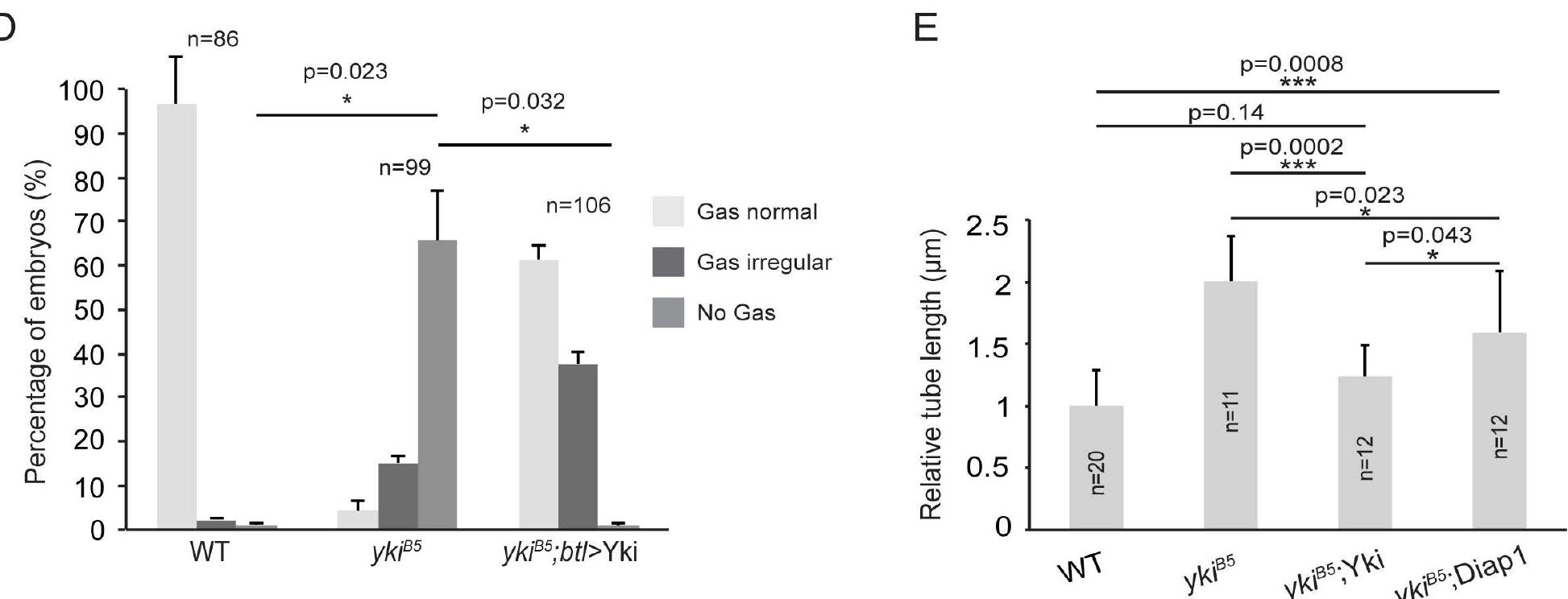

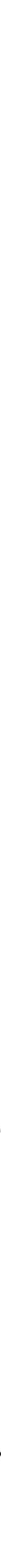
A

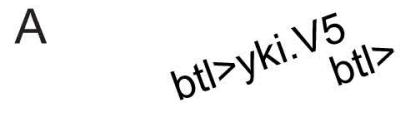

$20 \mathrm{kDa}-$

$15 \mathrm{kDa}-$

IP: a-V5, WB: $a-t s r$

$50 \mathrm{kDa}-$

IP: a-V5, WB: a-V5

$20 \mathrm{kDa}-$

D
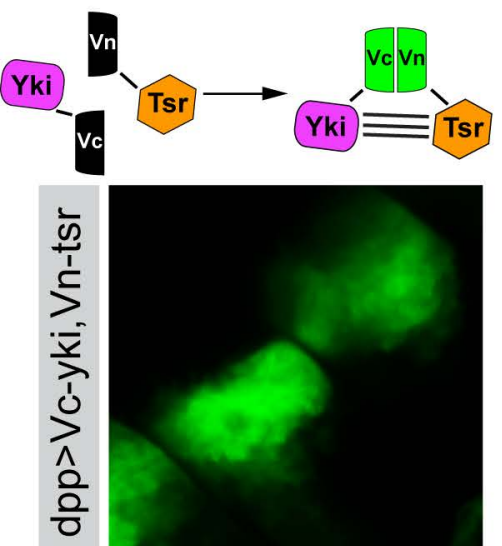
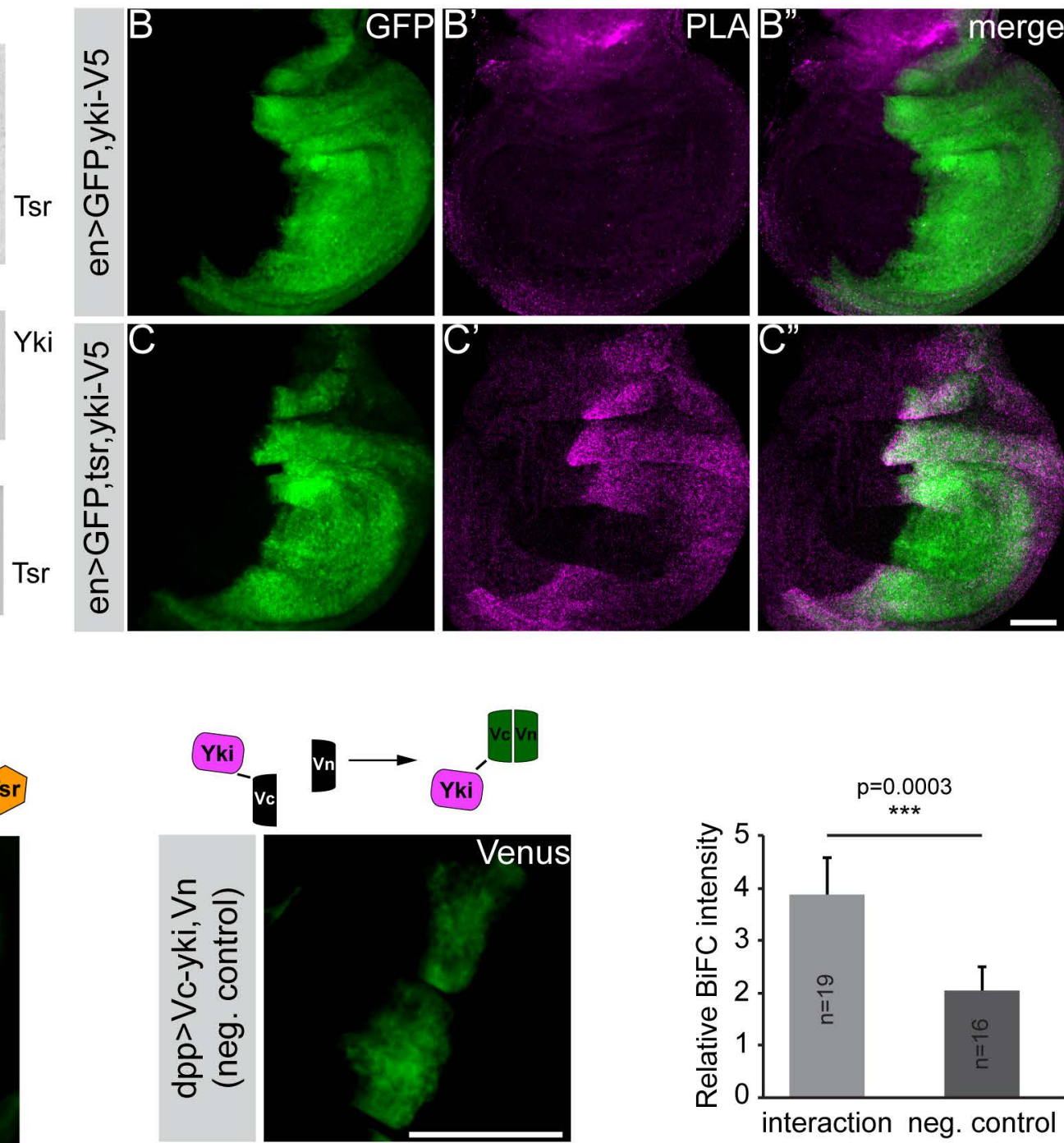

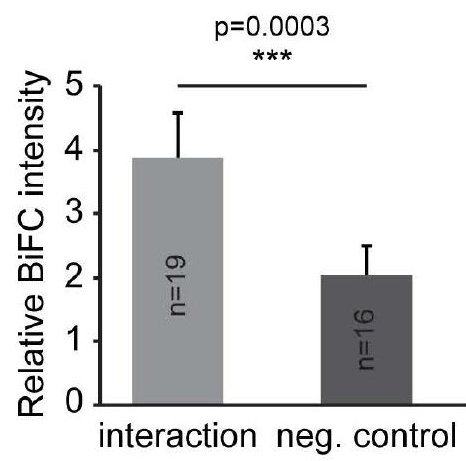



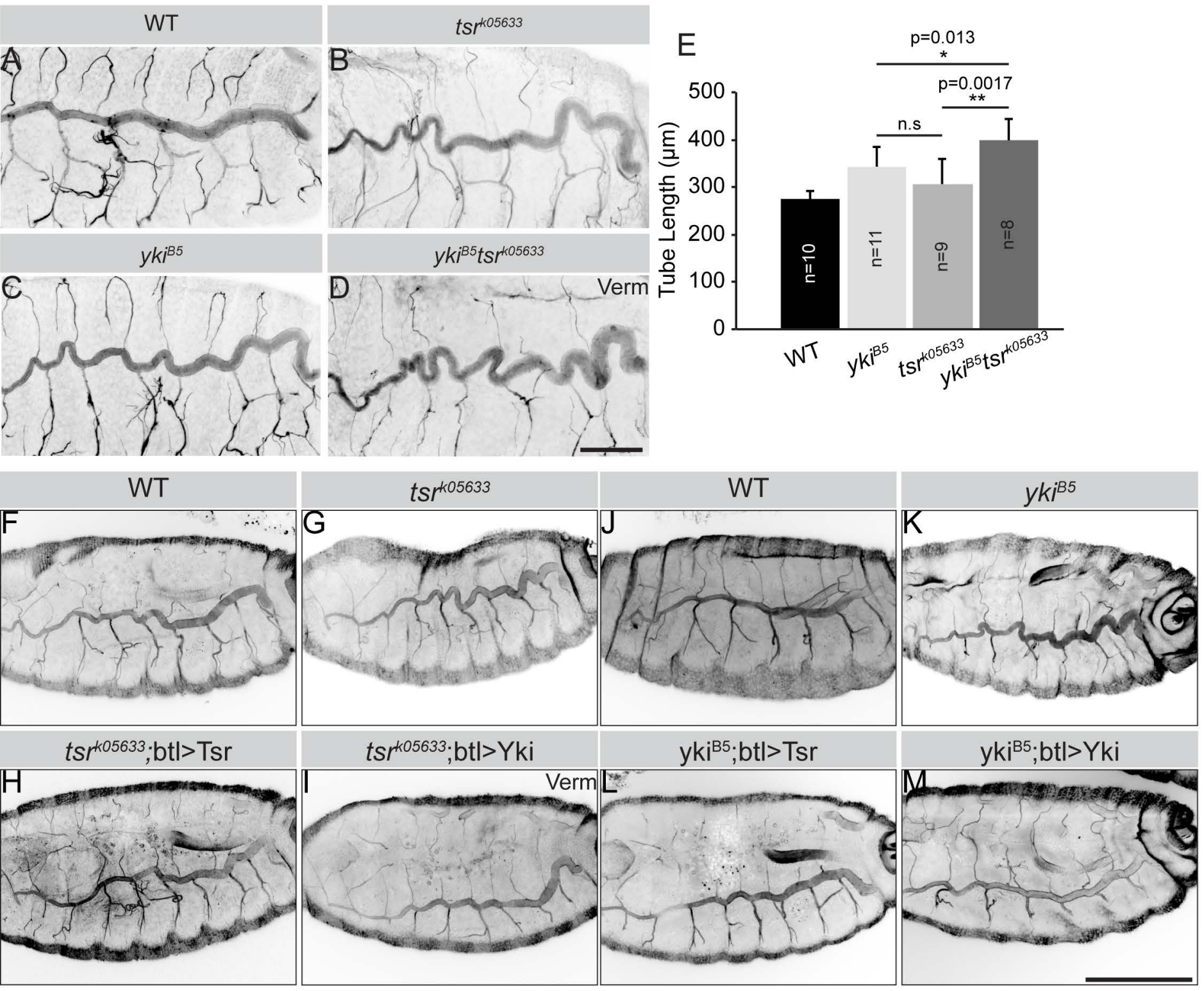

$\mathrm{N}$

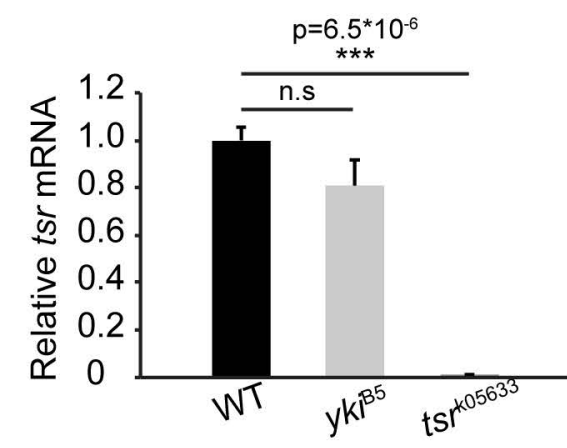

0

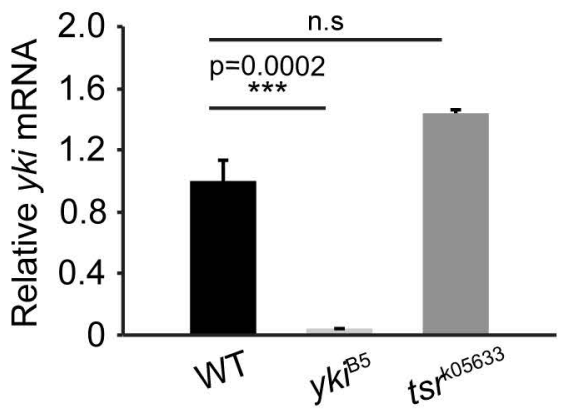

R

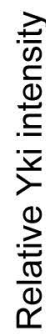

C

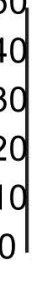

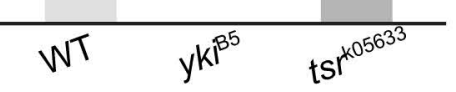

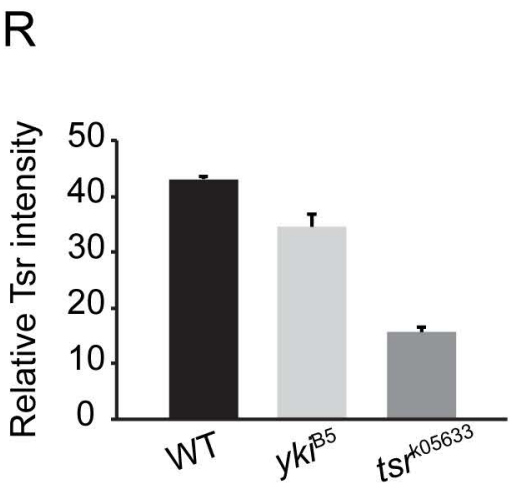

P

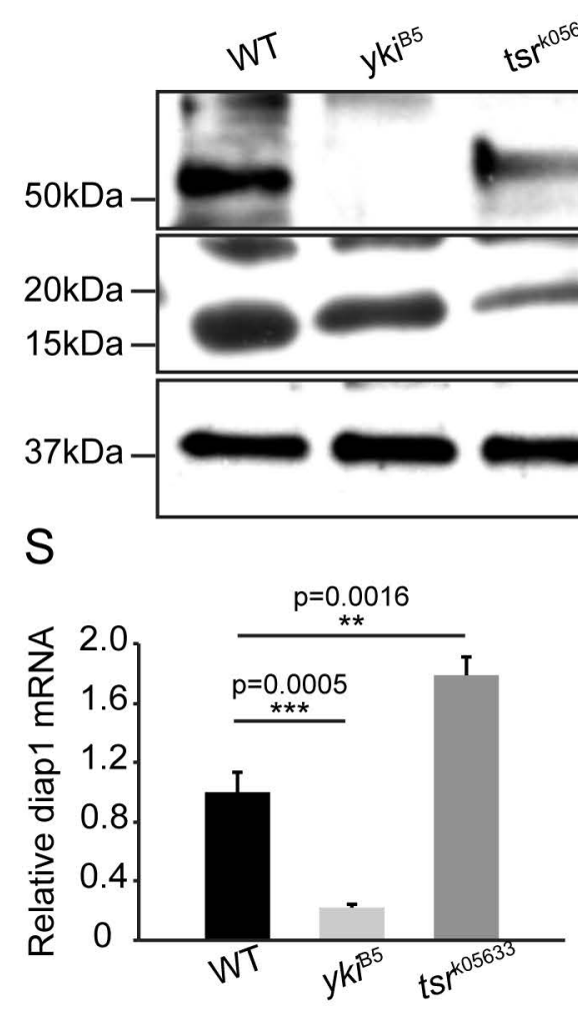



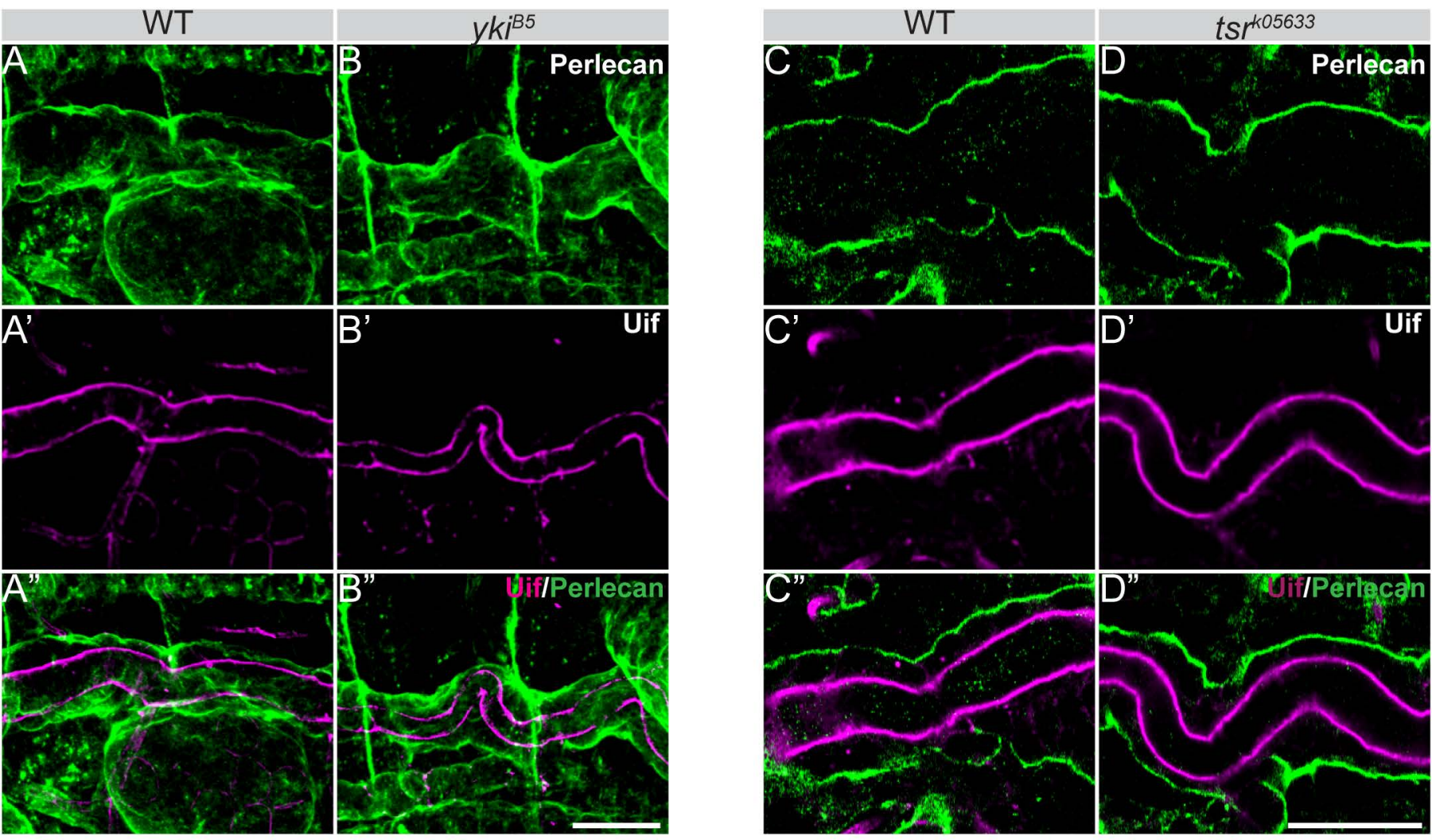
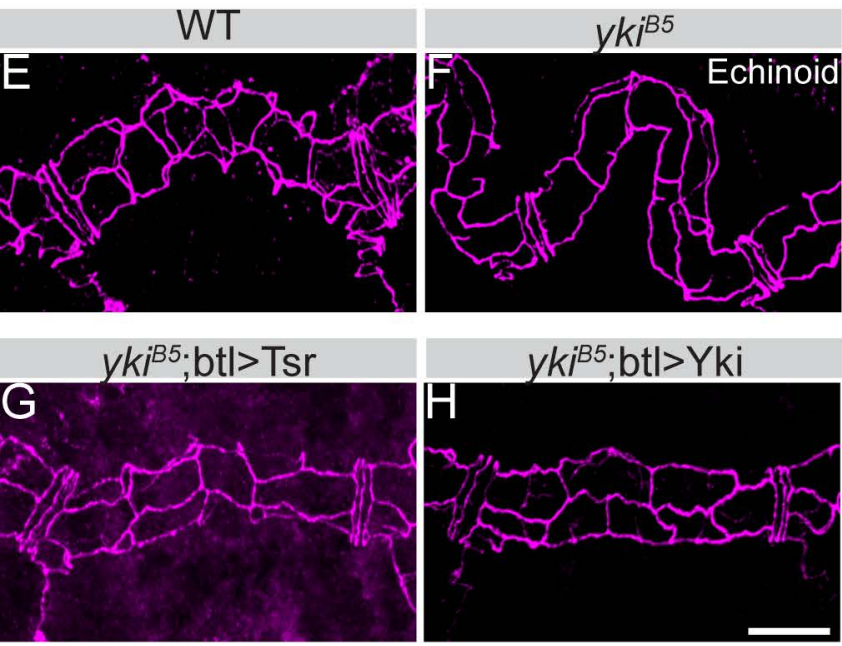

M

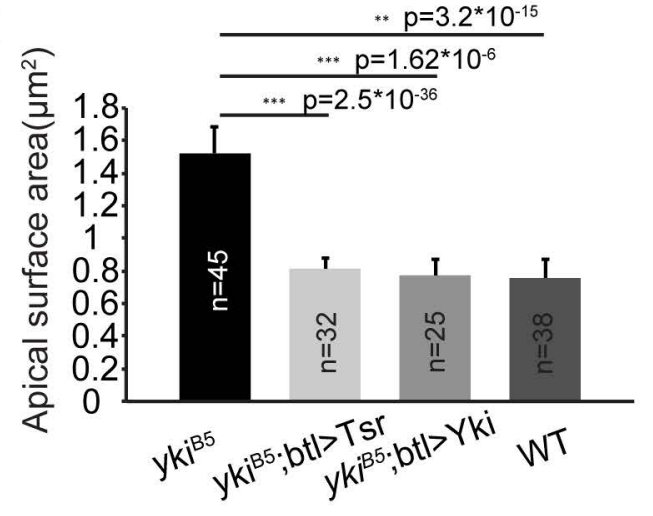

$y k^{B 5} ;$ btl>Yki

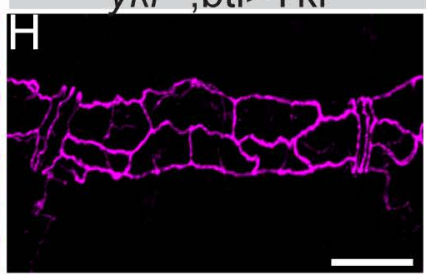

$* p=3.2 * 10^{-15}$

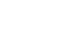

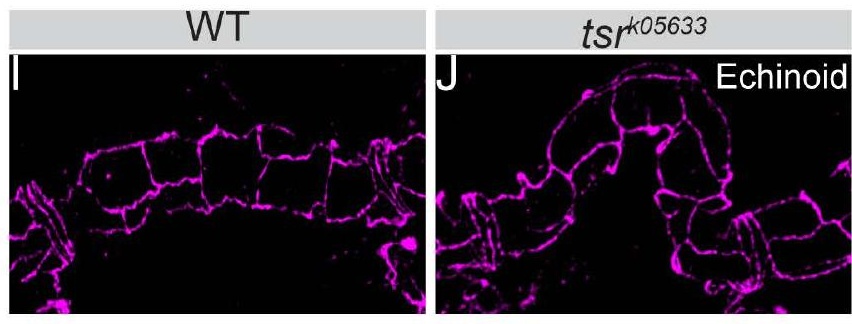
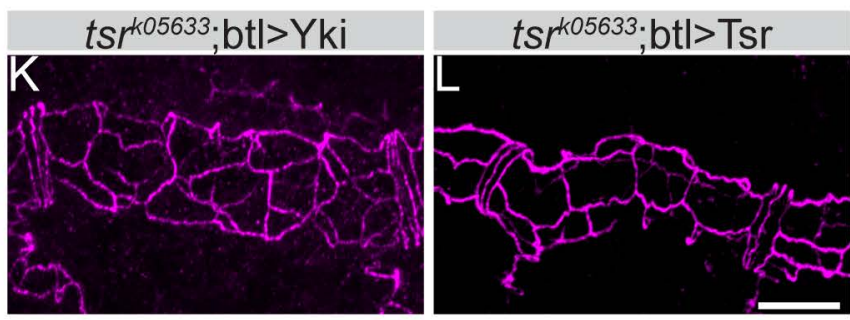

$\mathrm{N}$

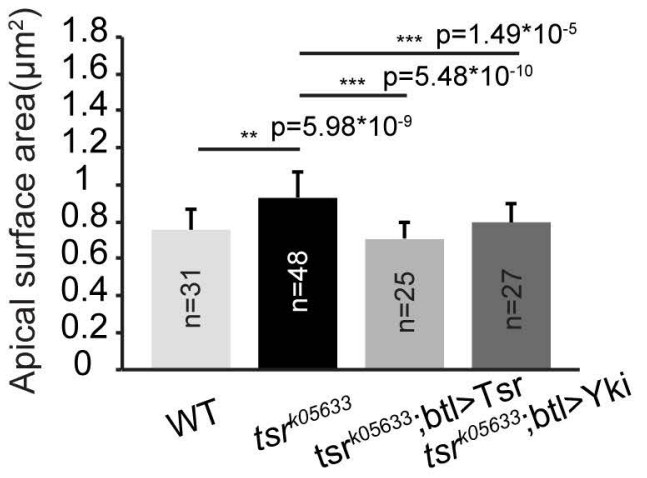


Figure 9

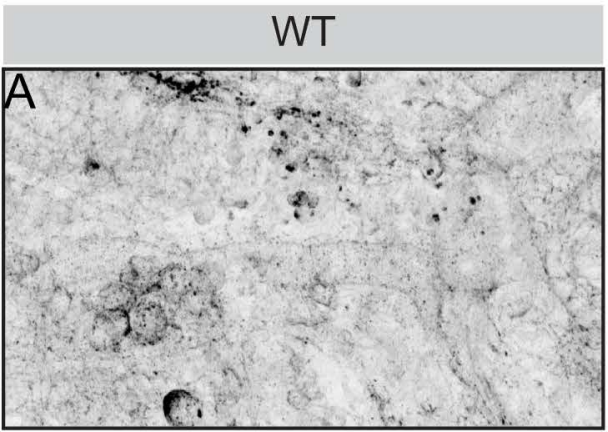

$t_{s} r^{k 05633}$

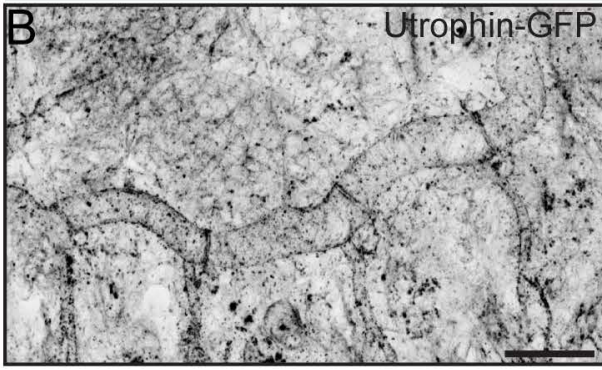

$\overline{A^{\prime}}$

WT
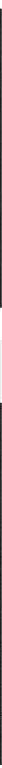

D. $F, F$ - Otrophin-GEP
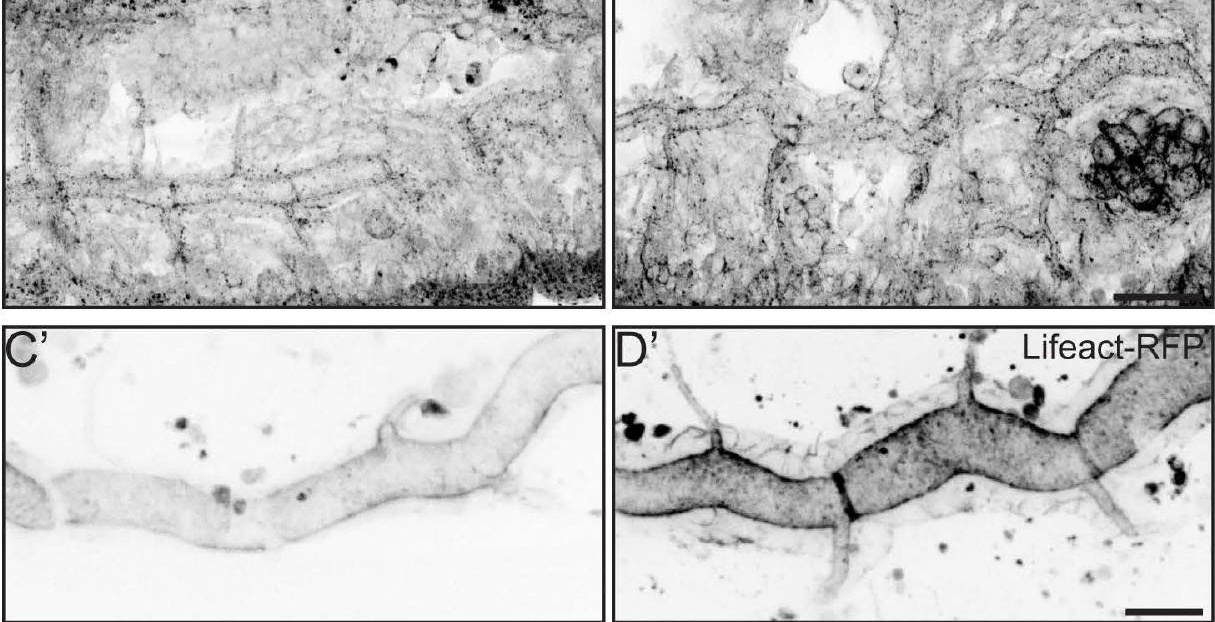
$A$

mKate-Yki
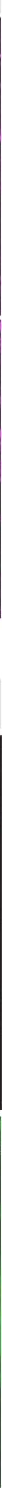

Figure 10

\section{WT}

$y k i^{B 5}$

$\sqrt{B}$

are 4.

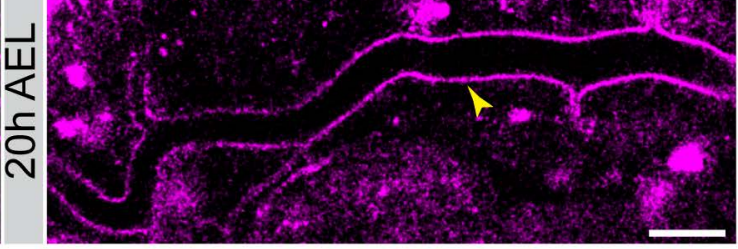

C $x^{2}=y^{2}+x^{2}+2 z^{2}$

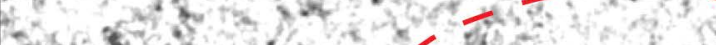




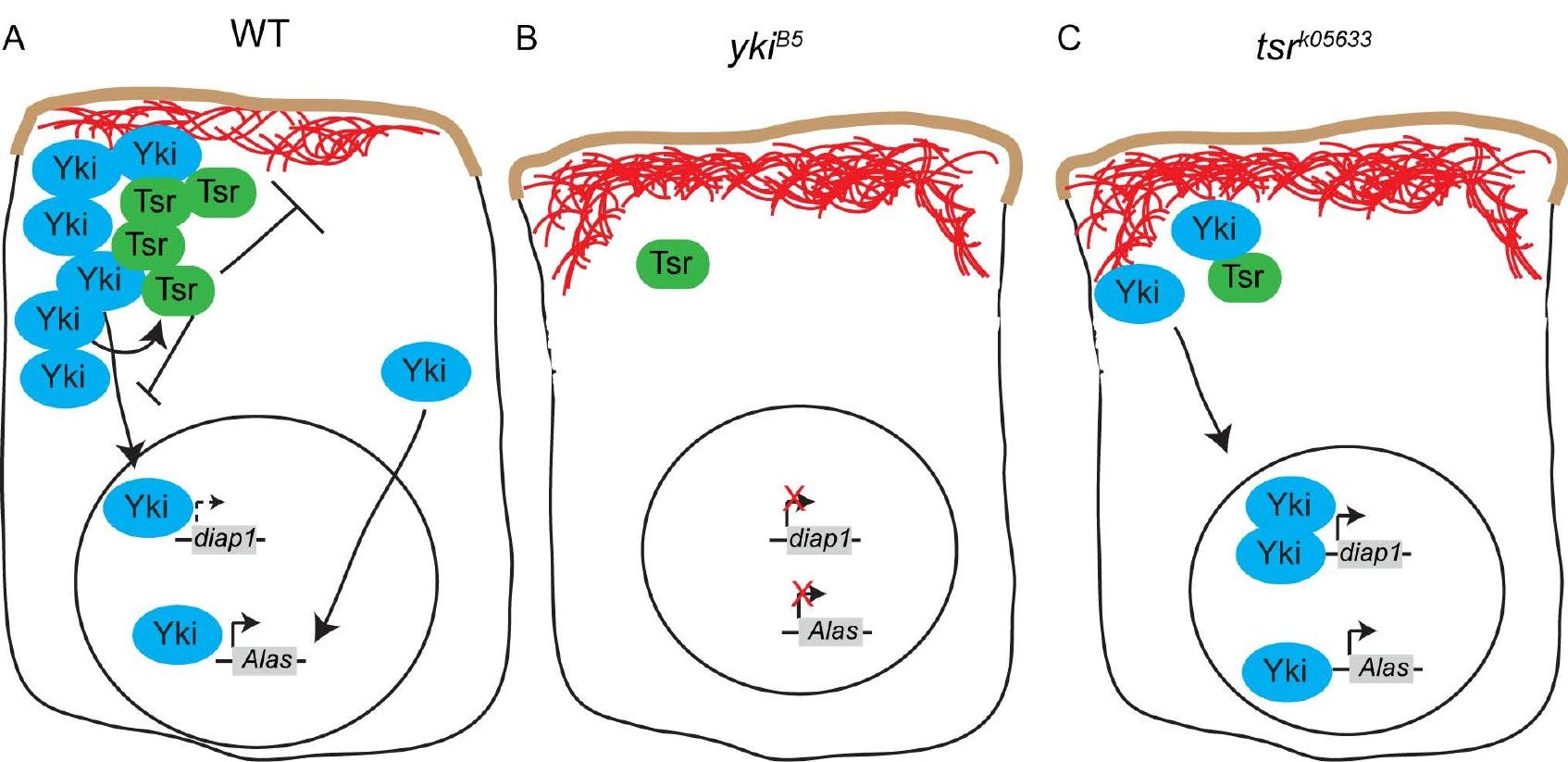


Supplemental Figure 1

SJ markers

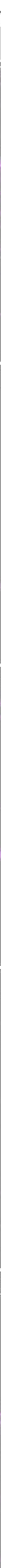


Supplemental Figure 2

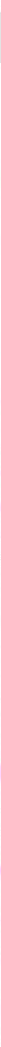




\section{Supplemental Figure 3}

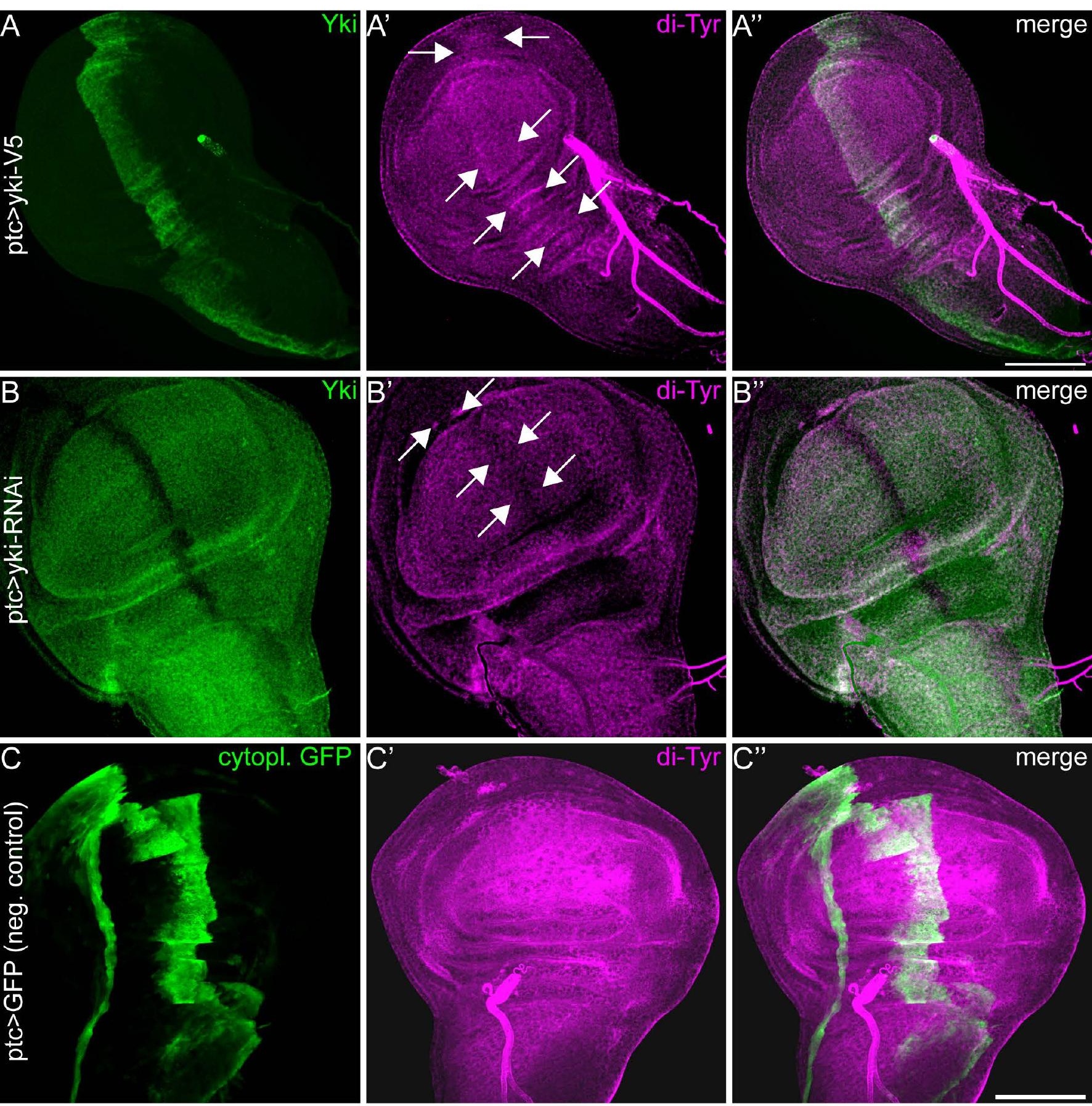

D

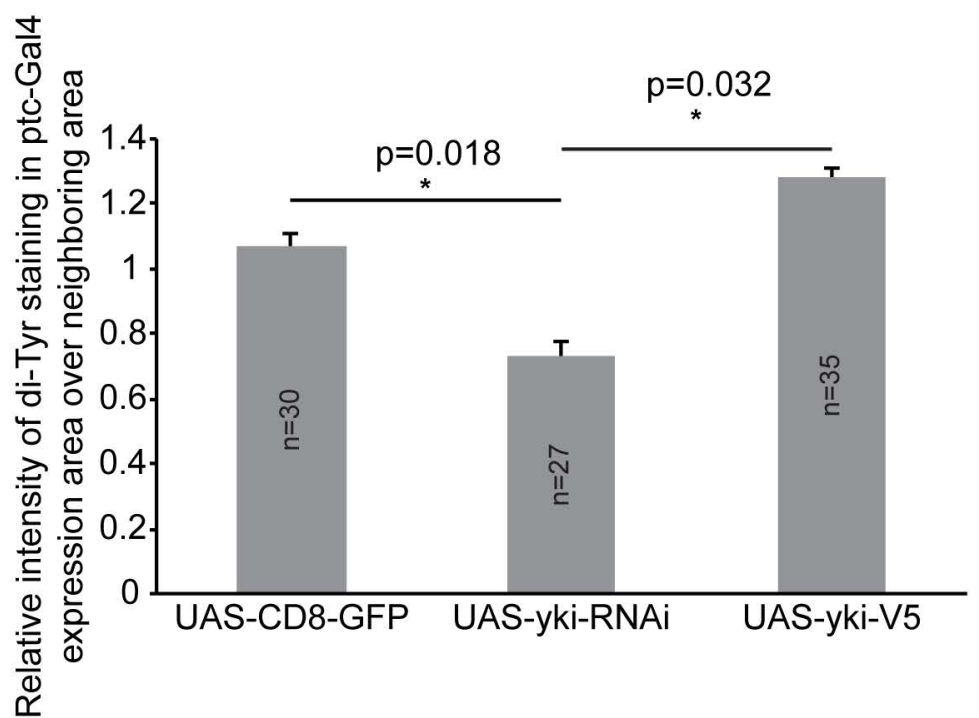


Supplemental Figure 5

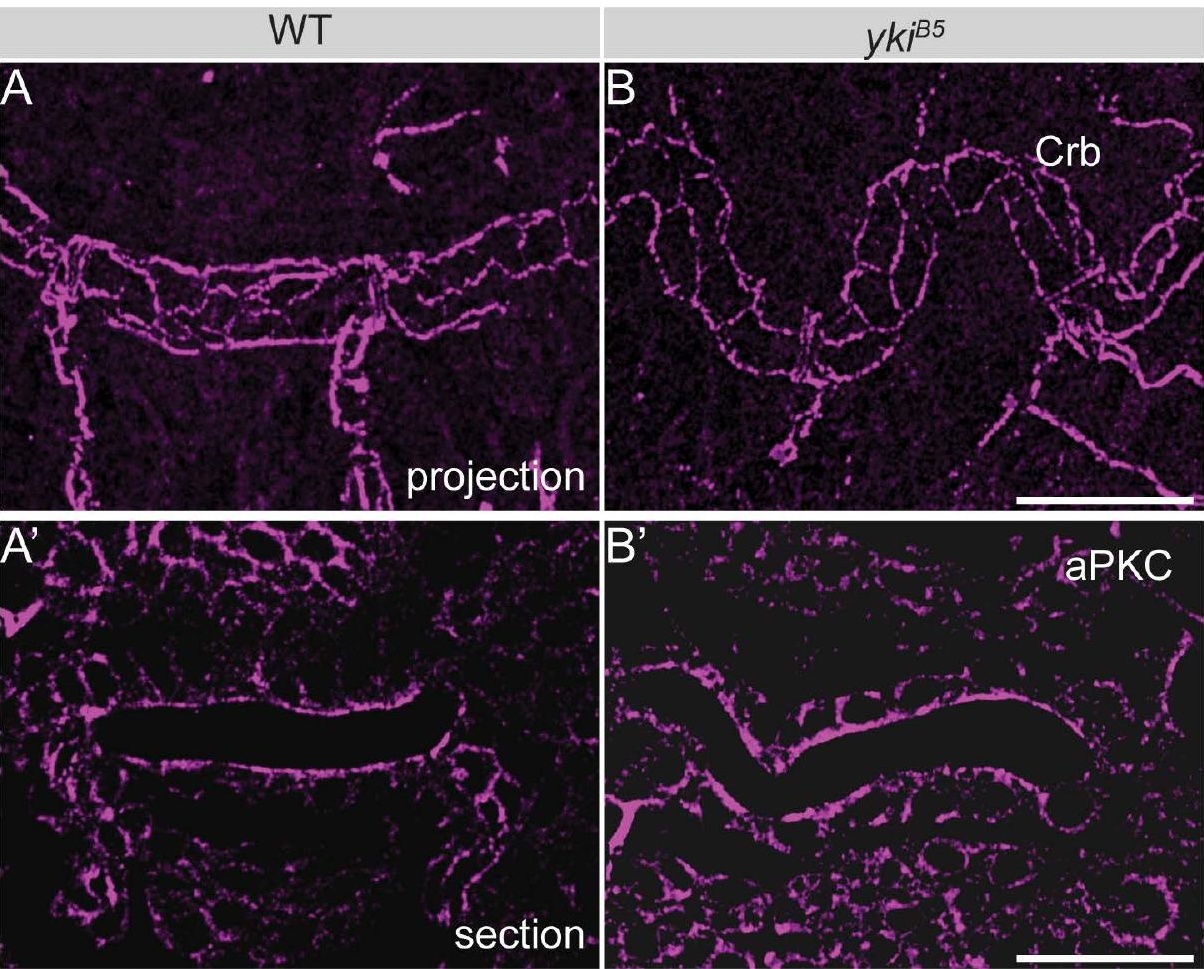


Supplemental Fig. 6

A

\begin{tabular}{|c|c|c|c|c|c|c|}
\hline \multirow{2}{*}{\multicolumn{2}{|c|}{ Experiment N }} & \multicolumn{2}{|c|}{$\begin{array}{l}\text { Bait (V5-tag Yorkie } \\
\text { gi221468529) }\end{array}$} & \multicolumn{3}{|c|}{ Twinstar (gi17136986) } \\
\hline & & $\begin{array}{l}\mathrm{N} \text { of unique } \\
\text { peptides }^{a}\end{array}$ & $\begin{array}{l}\mathrm{N} \text { of } \\
\text { spectra }^{a}\end{array}$ & $\begin{array}{l}\mathrm{N} \text { of unique } \\
\text { peptides }^{a}\end{array}$ & $\begin{array}{l}\mathrm{N} \text { of } \\
\text { spectra }^{a}\end{array}$ & Enrichment ${ }^{b}$ \\
\hline \multirow[t]{2}{*}{ I } & $\mathrm{IP}^{\mathrm{C}}$ & 15 & 99 & 2 & 9 & 1.8 \\
\hline & control $^{c}$ & n.a & n.a & 4 & 4 & \\
\hline \multirow[t]{2}{*}{ II } & $\mathrm{TPC}$ & 16 & 110 & 4 & 12 & 2.9 \\
\hline & control $^{C}$ & n.a & n.a & 4 & 7 & \\
\hline
\end{tabular}



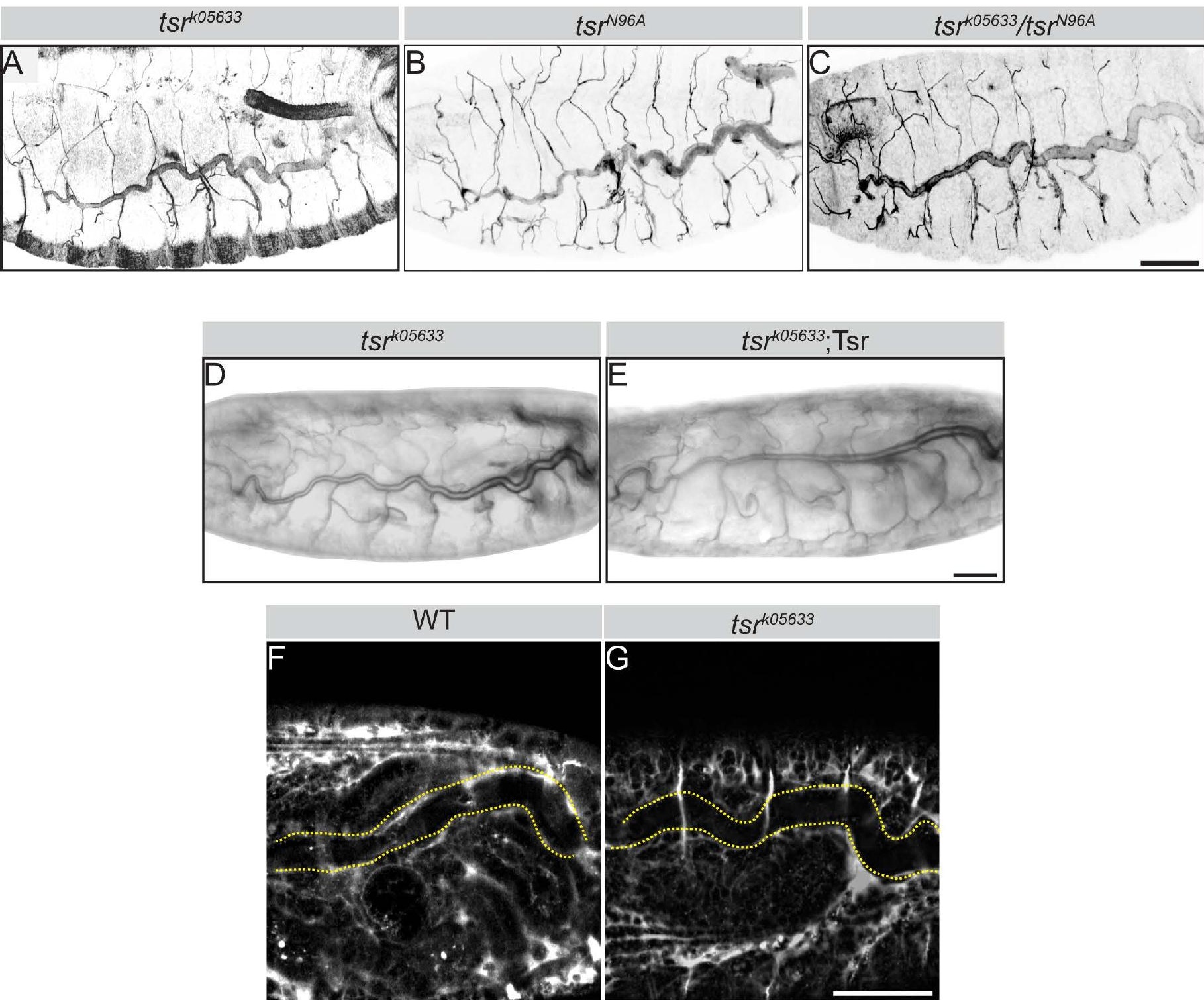
Supplemental Figure 8

A

B

embryos (unfertilized)
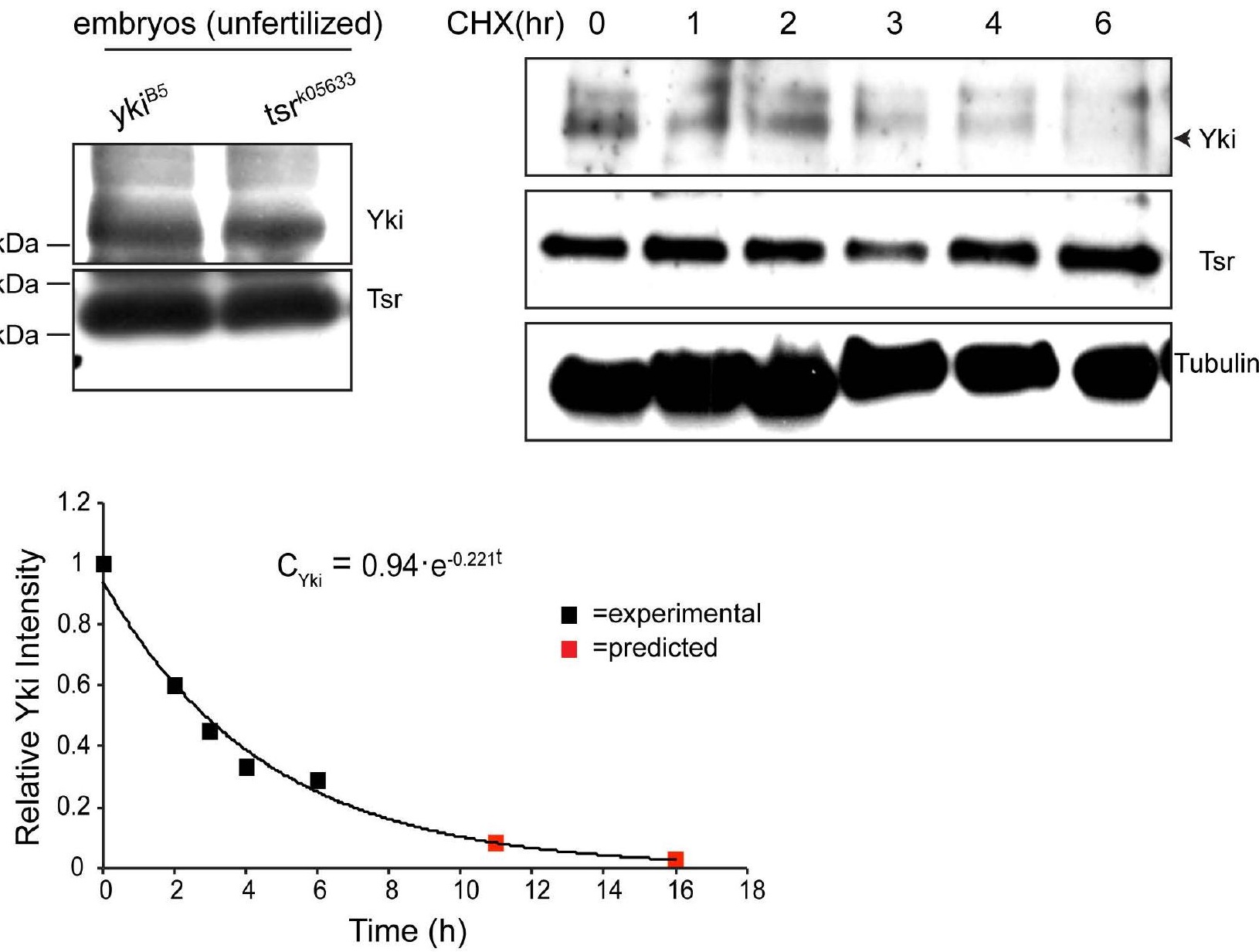


\section{GFP.Cofilin}

\section{+ siCofilin \\ +siluci}

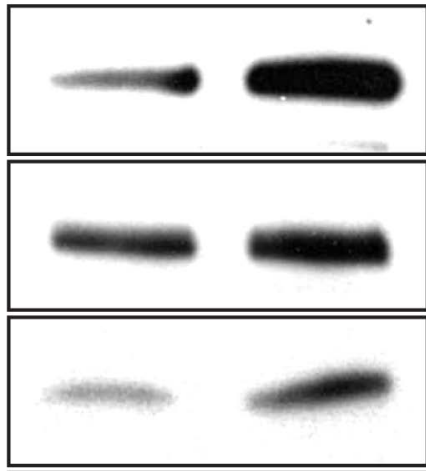

PYAPS381

PYAPS 127

\section{Total YAP}

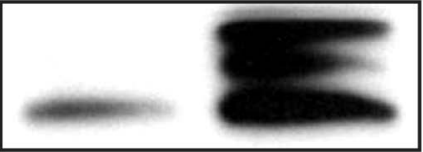

GFP.Cofilin

Cofilin

Tubulin 


\section{Supplemental Figure 10}

A

B

Hnt
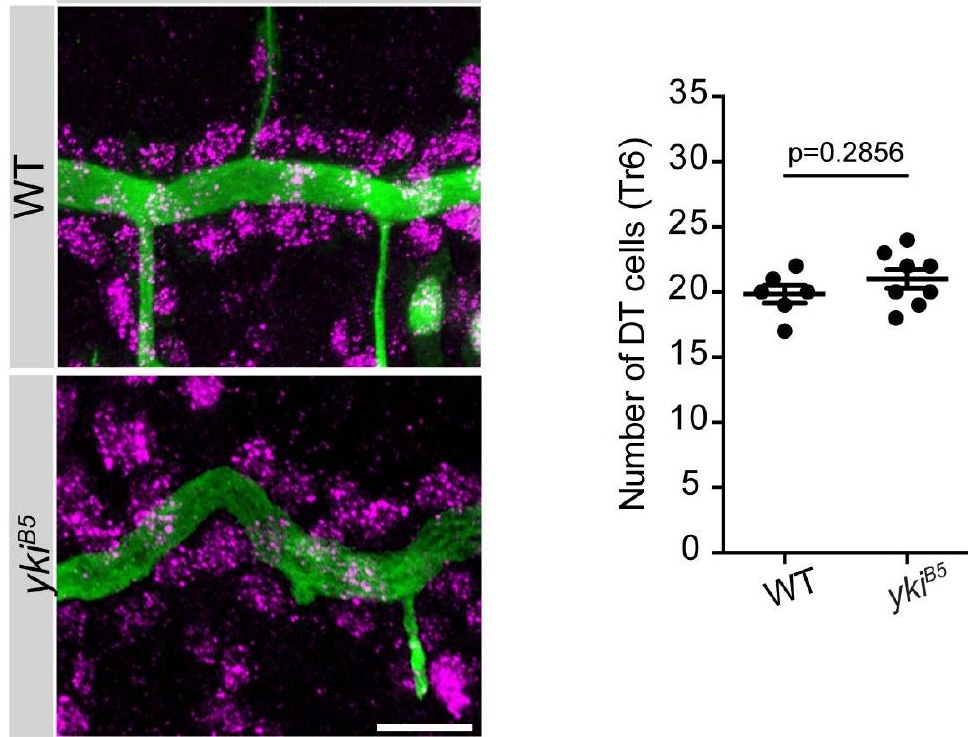


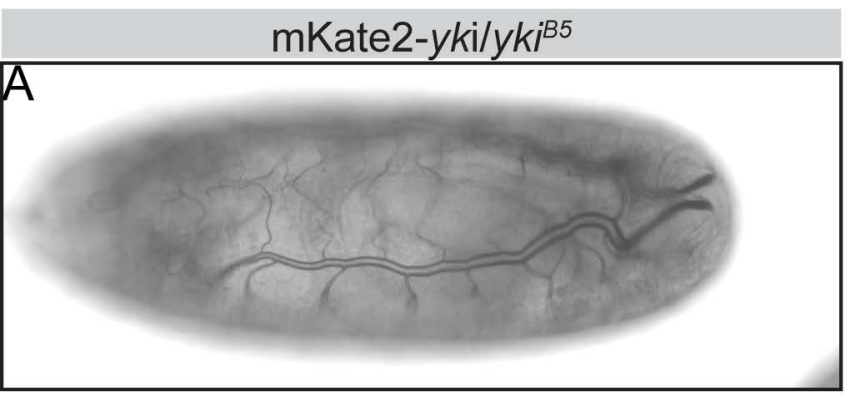

$\bar{B}$

mKate2-yki/mKate2-yki

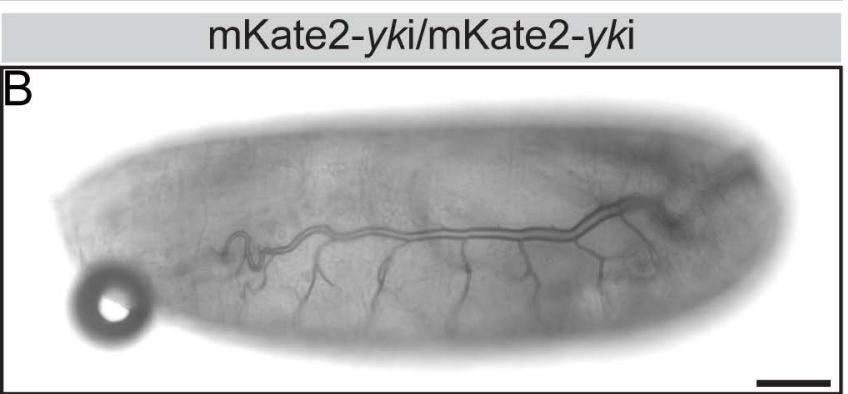

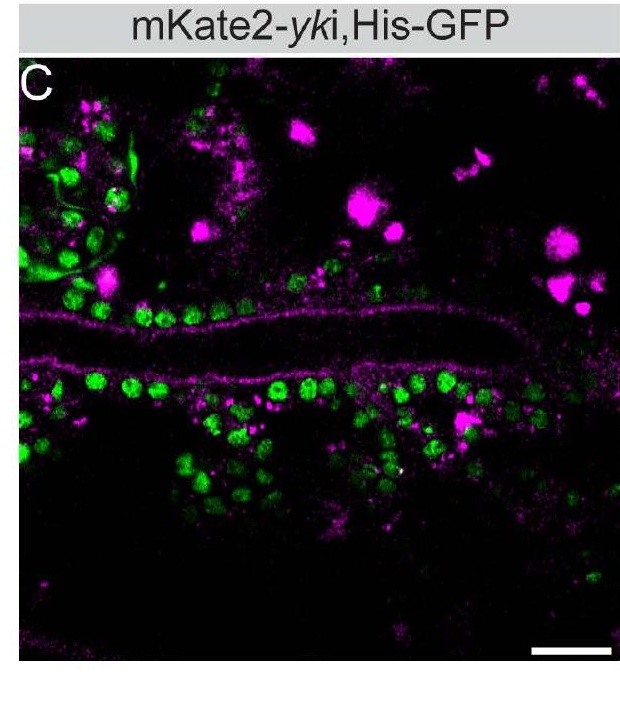

\section{mKate2-yki,His-GFP}

